\title{
Exploring anomeric glycosylation of phosphoric acid: optimisation and scope for non- native substrates
}

Laura Beswick, Sanaz Ahmadipour, Gert-Jan Hofman ${ }^{\perp}$, Hannah Wootton, Eleni Dimitriou, Jóhannes Reynisson $\$$, Robert A. Field`, Bruno Linclau ${ }^{\perp}$ and Gavin J. Miller*

Lennard-Jones Laboratory, School of Chemical and Physical Sciences, Keele University, Keele, Staffordshire ST5 5BG, United Kingdom

^Department of Chemistry and Manchester Institute of Biotechnology, The University of Manchester, 131 Princess Street, Manchester M1 7DN, United Kingdom

${ }^{\perp}$ School of Chemistry, University of Southampton, Highfield, Southampton SO17 1BJ, U.K.

$\$$ Hornbeam building, School of Pharmacy and Bioengineering, Keele University, Keele, Staffordshire, ST5 5BG, United Kingdom

Glycosyl 1-phosphates are key intermediates in carbohydrate primary metabolism and are utilised by microorganisms to form polyphosphate architectures that constitute keys parts of their extracellular capsule and cell walls. ${ }^{[1-4]}$ They serve as precursors to sugar nucleotides, ${ }^{[5-6]}$ the sugar donor components utilised by glycosyltransferases in the assembly of oligosaccharides and glycans and have played a key role in the development of glycosylated natural-product-based therapeutics. ${ }^{[7]}$ Additionally, glycosyl 1-phosphates have been used as substrates for glycoside phosphorylases, a rapidly expanding ${ }^{[8]}$ family of CAZy enzymes for the synthesis of oligosaccharide targets ${ }^{[9]}$ and also play important technological roles in the food and detergent sectors. ${ }^{[10-11]}$

In order to access these significant materials, a variety of chemical and chemoenzymatic strategies have been developed. Chemoenzymatic methods frequently involve glycosyl kinases ${ }^{[12-15]}$ although phosphomutase enzymes have also been explored. ${ }^{[16]}$ From a chemical perspective, several synthetic options exist to create glycosyl 1-phosphates, most commonly via anomeric glycosylation or hemi-acetal deprotonation and reaction with a suitable phosphorous electrophile. ${ }^{[2,17]}$ These approaches both present a capability to modify native glycosyl 1-phosphate structures, enabling to then interrogate the biosynthetic enzymes and processes that utilise them. ${ }^{[18]}$

As part of a program pursuing the synthesis of glycosyl 1-phosphate and sugar nucleotide chemical tools, ${ }^{[19-21]}$ the MacDonald method for accessing anomeric 1-phosphates became of interest. This method has been successfully used by several groups, including for non-native systems. ${ }^{[22-27]}$ Originally published in $1962,{ }^{[28]}$ the procedure uses elevated temperature and low pressure to form a melt of crystalline phosphoric acid and a peracetylated sugar, glycosylating the anomeric position and releasing AcOH. This is followed by ester hydrolysis in the same pot to deliver the deprotected 1-phosphate. (Scheme 1). However, the reaction can be low yielding, requires a significant excess of $\mathrm{H}_{3} \mathrm{PO}_{4}(10$ equiv.) and purification of the product(s) is not facile. The capabilities of this transformation as a simple method for accessing modified 1-phosphates, quickly, from acetylated precursors required investigation to optimise the reaction and explore its scope further. 


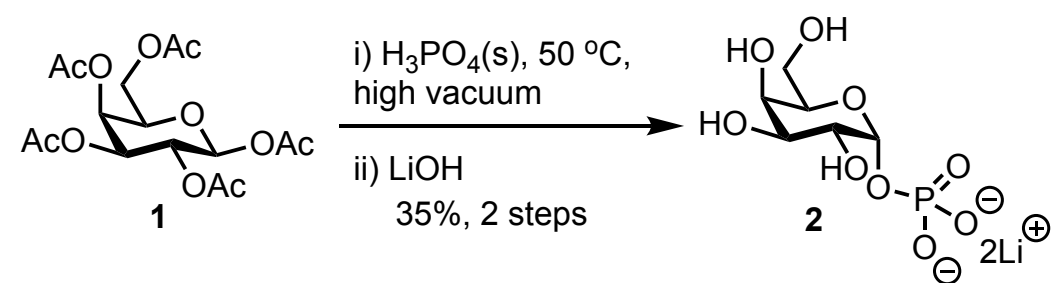

Scheme 1. Original MacDonald phosphorylation conditions to access D-Gal 1-phosphate 2 from per-acetylated precursor 1.

The crystalline phosphoric acid reagent is extremely hygroscopic and whilst received from suppliers as an opaque, crystalline solid, it readily forms a paste as hydration upon opening the container to the atmosphere is unavoidable. This is problematic for the ensuing reaction as water can compete with phosphate in the anomeric substitution reaction, which produces the corresponding hemi-acetal by-product, reducing the final yield.

In order to avoid this, a glove box (or commercially available glove bag) was used for the anomeric phosphorylation experiments. Hence, ten equivalents of phosphoric acid and $500 \mathrm{mg}$ (one equivalent) of $\mathbf{1}$ were transferred to a Schlenk tube under an atmosphere of nitrogen. The closed tube containing the reactants was then transferred to a dual line manifold and the solid mixture heated to a melt $\left(50{ }^{\circ} \mathrm{C}\right)$ under vacuum $($ Scheme 2$)$. Once the reaction was complete, as monitored by TLC analysis $(3 \mathrm{~h})$, the acetyl groups were saponified in the same pot and, following work-up, $\mathbf{2}$ was isolated as a white solid. Examination of $\mathbf{2}$ by ${ }^{1} \mathrm{H}$ and ${ }^{31} \mathrm{P}$ NMR showed $>90 \%$ conversion to the desired 1-phosphate: ${ }^{1} \mathrm{H} \delta 5.31$ (dd, $J=7.3$, $\left.3.6 \mathrm{~Hz}, \mathrm{H}_{1}\right)$. The only impurity observed was a trace amount of the hemi-acetal byproduct $(<10 \%)$. As necessary, this could easily be removed using a strong anion exchange column to elute the uncharged species with water, followed by an ammonium bicarbonate eluent to release $\mathbf{2}$ in a much improved 68\% final yield (after freeze-drying), compared to the original procedure.
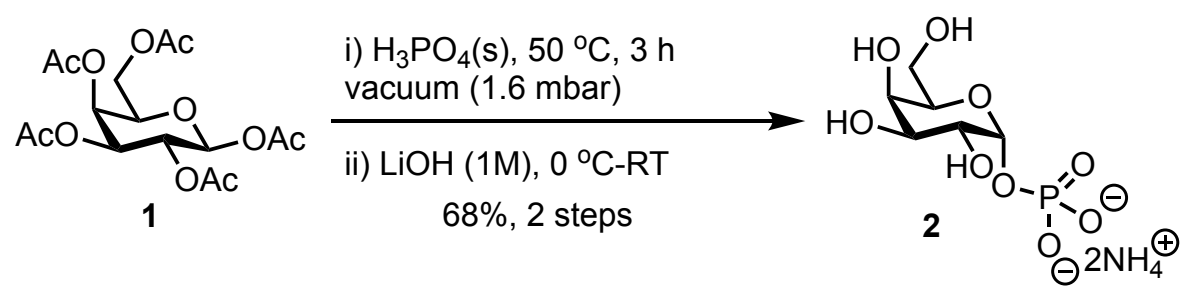

Scheme 2. Phosphorylation of per-acetylated D-Gal 1.

Encouraged by these improvements to the yield and purity of the reaction outcome, other reaction parameters in the conversion of $\mathbf{1}$ to $\mathbf{2}$ were targeted. These findings are summarised in Table 1.

Table 1. Optimisation of per-acetyl D-Gal anomeric phosphorylation

\begin{tabular}{|c|c|c|c|c|c|}
\hline \multicolumn{2}{|c|}{$\underbrace{O A c O}_{\text {OAc }}$} & \multicolumn{4}{|c|}{$\underset{\text { ii) } \mathrm{LiOH}(1 \mathrm{M}), 0^{\circ} \mathrm{C}-\mathrm{RT}}{\longrightarrow}$} \\
\hline Entry & $\begin{array}{c}\text { Equivalents } \\
\mathrm{H}_{3} \mathrm{PO}_{4}\end{array}$ & $\begin{array}{c}\text { Temperature } \\
\left({ }^{\circ} \mathrm{C}\right)^{\#}\end{array}$ & $\begin{array}{l}\text { Yield } \\
(\%)\end{array}$ & $\begin{array}{l}\text { Scale } \\
(\mathrm{mg} \mathrm{1)})\end{array}$ & $\begin{array}{l}\text { Time } \\
\text { (h) }\end{array}$ \\
\hline 1 & 10.0 & 50 & 68 & 500 & 3 \\
\hline 2 & 10.0 & 50 & 65 & 100 & 3 \\
\hline 3 & 10.0 & 50 & 69 & 50 & 3 \\
\hline
\end{tabular}




\begin{tabular}{|c|c|c|c|c|c|}
\hline 4 & 10.0 & 40 & 62 & 80 & 3 \\
\hline 5 & 5.0 & 40 & 67 & 300 & 3 \\
\hline 6 & 5.0 & 35 & 49 & 200 & 3 \\
\hline
\end{tabular}

"Temperature of the heating block, not the internal reaction temperature.

The initial reaction with commercially available 1 was conducted on $500 \mathrm{mg}$ scale. However, for more exotic, non-native substrates, material availability is often a limiting factor and so a reduction in the scale of the reactions, down to ranges between 50 and 100 $\mathrm{mg}$, was investigated first. Pleasingly, little effect was observed on the isolated yield (Table 1, entries 2 and 3). Generally, this phosphorylation is carried out at a temperature ranging between 50 and $60{ }^{\circ} \mathrm{C}$ (to form the melt and assist in removing $\mathrm{AcOH}$ under vacuum). The reaction was conducted successfully at $40{ }^{\circ} \mathrm{C}$ (Table 1 , entry 4 ) using a high vacuum line pressure of $1.6 \mathrm{mbar}$, also noting that the required melt failed to form efficiently when the temperature was lowered further to $30{ }^{\circ} \mathrm{C}$. Finally, the equivalents of phosphoric acid required was successfully reduced from ten to five (Table 1, entries 5 and 6), observing this to strike the best balance between reaction time (increasing this led to blackening of the melt and thus reduced yields) and full conversion of starting material by TLC at $40{ }^{\circ} \mathrm{C}$. With an improved procedure for this phosphorylation in hand a series of monosaccharide substrates was selected for evaluation. The results of these experiments are presented in Table 2 and discussed thereafter.

Table 2. Exploring substrate scope for anomeric phosphorylation

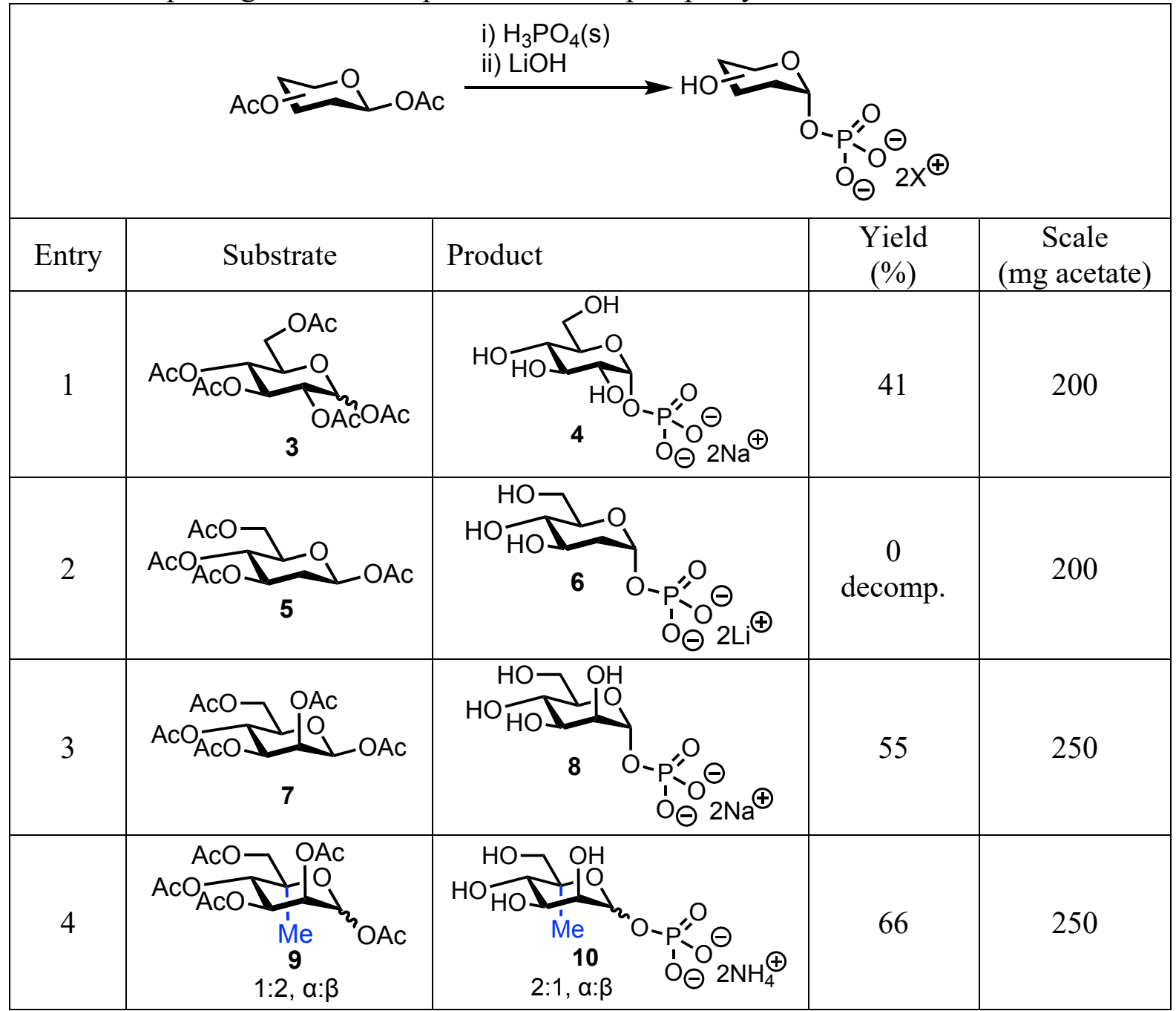




\begin{tabular}{|c|c|c|c|c|}
\hline 5 & ${ }_{11}^{\mathrm{AcO}} \underset{\mathrm{AcO}}{\mathrm{OAc}} \mathrm{OAC}$ & 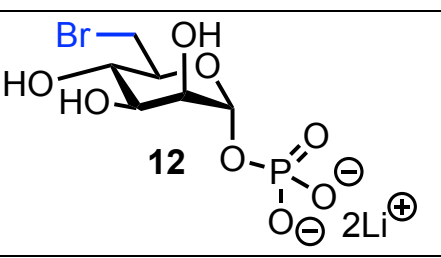 & $0\left(85^{\mathfrak{f}}\right)$ & 400 \\
\hline 6 & ${ }_{14}^{A c O}$ & $15{ }^{O-P_{1}^{\prime \prime}}$ & $72 *$ & 54 \\
\hline 7 & $\underbrace{}_{16} \underbrace{O A c}_{A c C}$ & 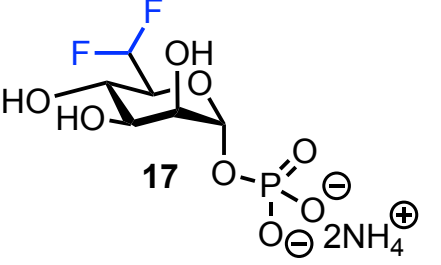 & 42 & 45 \\
\hline 8 & ${ }_{18}^{\mathrm{AcO}_{\mathrm{AcO}}} \underbrace{\mathrm{OAc}}_{\mathrm{OAC}}$ & 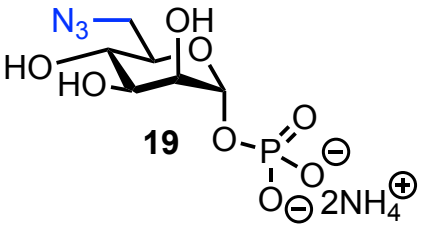 & 65 & 100 \\
\hline 9 & ${ }_{20}^{\mathrm{AcS}}{ }_{\mathrm{AcO} \perp}^{\mathrm{OAc}}$ & 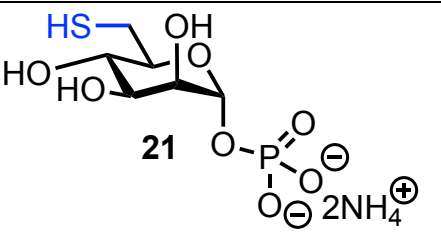 & $43^{\$}$ & 100 \\
\hline
\end{tabular}

Conditions used were initially the same as Table 1, entry 5. ${ }^{£}$ See discussion for formation of cyclic product 13 below. * formed as a 1:0.3 mixture with 13. ${ }^{\$}$ Observed as the disulfide.

For per-acetyl-D-glucose 3, anomeric phosphorylation proceeded smoothly, and the target 1-phosphate 4 was isolated in $41 \%$ yield (Table 2, entry 1). This was lower than observed for 2 and was attributed to the melt requiring a higher temperature $\left(60^{\circ} \mathrm{C}\right)$ to effect full conversion, which led to a blackening of the reaction mixture. The reaction for 2-deoxy glucose derivative 5 showed only decomposition by TLC after $3 \mathrm{~h}$ at a temperature of $60{ }^{\circ} \mathrm{C}$, with the reaction melt turning black almost immediately (Table 2, entry 2). After several repeats, no conversion into $\mathbf{6}$ was detected, concluding 5 to be a poor substrate for these anomeric phosphorylation conditions. Finally, for these simple glycosyl 1-phosphates the Dmanno compound $\mathbf{8}$ was isolated in satisfactory $55 \%$ yield from 7 , noting that the reaction temperature was successfully lowered to $45^{\circ} \mathrm{C}$ (Table 2, entry 3 ).

A 5-C-methyl mannose derivative was evaluated next to establish if a steric effect from a C5-axial methyl group would influence the anomeric selectivity of phosphorylation. Following the procedure reported by Davis et al, ${ }^{[29]} 5-C$-methyl-D-mannose was accessed and acetylation of the free sugar using either $\mathrm{Ac}_{2} \mathrm{O}$ /pyridine or $\mathrm{Ac}_{2} \mathrm{O} / \mathrm{H}_{2} \mathrm{SO}_{4}$ gave 9. Anomeric phosphorylation was accomplished in good yield (66\%) to deliver 10. (Table 2, Entry 4). Formation of an $\alpha / \beta$ 1-phosphate mixture was observed by ${ }^{1} \mathrm{H}$ NMR $\left[{ }^{1} \mathrm{H} \delta 5.27(\mathrm{dd}, J=9.1\right.$, $\left.\left.2.2 \mathrm{~Hz}, \mathrm{H}_{1 \alpha}\right), 5.19\left(\mathrm{dd}, J=8.6,1.1 \mathrm{~Hz}, \mathrm{H}_{1 \beta}\right)\right]$ with a $2: 1$ preference for formation of the $\alpha-$ anomer. This finding indicated that a sterically encumbering $\mathrm{C} 5-\mathrm{Me}$ group introduced a competing pathway for formation of the $\beta$ 1-phosphate. To question whether the formation of 10 was influenced by the ratio of anomeric mixture used as starting material the ratio of $\alpha$ acetate in 9 was increased to $50 \%$ from $33 \%$ (accomplished by acetylation with $\mathrm{Ac}_{2} \mathrm{O} / \mathrm{H}_{2} \mathrm{SO}_{4}$ instead of $\mathrm{Ac}_{2} \mathrm{O} / \mathrm{Pyr}$ ). However, this made only a small difference to the observed $\alpha / \beta$ ratios 
in the product ( $\alpha / \beta$-10 2:1, from a $1: 2 \alpha / \beta$ ratio in 9 and $\alpha / \beta-102.5: 1$, from a $1: 1 \alpha / \beta$ ratio in 9).

A series of C6-halogenated analogues (Table 2, entries 5-7) were targeted next. Starting from C6-bromide 11 (accessed via an Appel reaction from the corresponding C6alcohol $\left.{ }^{[30]}\right)$, exposure to the established conditions showed a smooth conversion to one product in $85 \%$ yield, which is proposed to be cyclic phosphate 13, not the expected 1phosphate 12 (Scheme 3). HRMS analysis for 13 showed no bromine isotope pattern and inspection of the ${ }^{1} \mathrm{H}$ NMR data showed an apparent triplet in the anomeric region $[\delta 5.20$ (app. t, $J=6.9 \mathrm{~Hz}$ )]. This indicated that the ${ }^{3} J_{\mathrm{P}-\mathrm{H} 1}$ and ${ }^{3} J_{\mathrm{H} 2-\mathrm{H} 1}$ coupling constants were now almost equal and distinct from the characteristic doublet of doublets for $\mathrm{H}_{1}$ in a 1-phosphate e.g. as seen for $8\left[\delta 5.23\left(\mathrm{dd}, J=8.6 \mathrm{P}-\mathrm{H} 1,1.3_{\mathrm{H} 1-\mathrm{H} 2} \mathrm{~Hz}\right]\right.$. In addition, the apparent triplet usually observed for $\mathrm{H}_{4}$ of ${ }^{4} \mathrm{C}_{1}$ mannose derivatives was now a doublet of doublets $[\delta 4.20(\mathrm{dd}, J=$ $6.0,2.8 \mathrm{~Hz}$ )], leading us to conclude that $\mathbf{1 3}$ had adopted an alternative solution state conformation. Inspection of the data for $\mathrm{H}_{6}$ showed the distinctive doublet of doublets to be absent for one of these protons as only the diastereotopic ${ }^{2} J$ coupling was observed $[\delta 4.10(\mathrm{~d}$, $J=10.9 \mathrm{~Hz}, \mathrm{H}_{6 e x o}$ ). A very small (not always detectable) ${ }^{3} J \mathrm{H}_{6-5}$ coupling exists only as the dihedral angle between these two protons approaches $90^{\circ}$, which can occur in a locked bicyclic system. Finally, ${ }^{31} \mathrm{P}_{-}{ }^{1} \mathrm{H}$ HMBC data for 13 demonstrated ${ }^{3} J_{\mathrm{P}, \mathrm{H}}$ coupling to both $\mathrm{H}_{1}$ and $\mathrm{H}_{6 e x o}$, confirming cyclisation through $\mathrm{C} 1 / \mathrm{C} 6$.
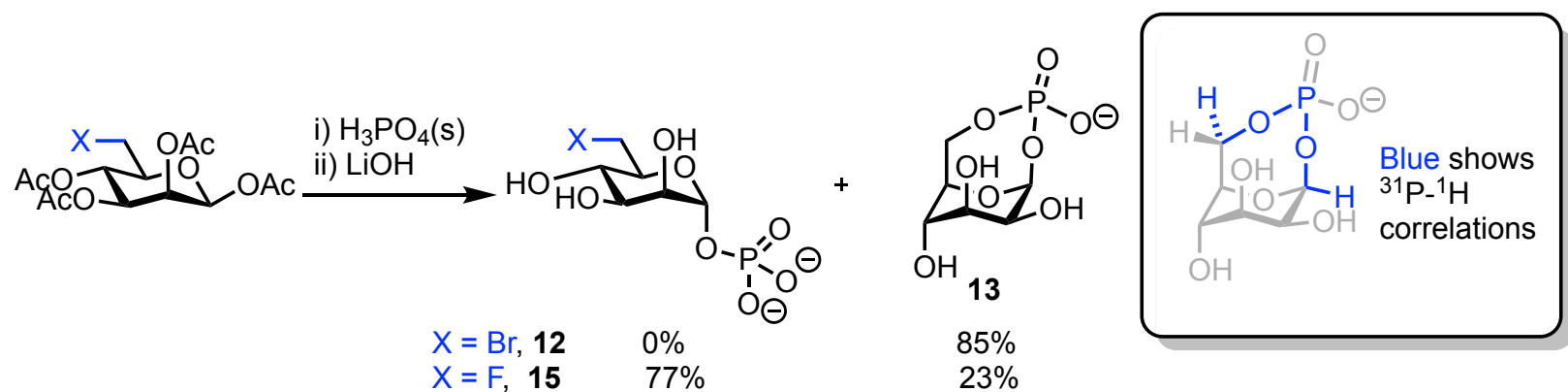

Scheme 3. Attempted phosphorylation of C6-halo D-mannose derivatives. Anomeric configuration of $\mathbf{1 3}$ is tentatively assigned as the $\beta$-configuration.

In accounting for this unexpected finding, it can be considered that phosphorylation at $\mathrm{C} 1$ of $\mathbf{1 1}$ occurred, followed by an intramolecular substitution of the C6 bromo derivative through anomeric phosphate oxygen (Scheme 4a). However, in the absence of X-ray crystallographic data the anomeric configuration of $\mathbf{1 3}$ is unconfirmed. As evidenced by the examples highlighted in Table 2, $\alpha$-phosphorylation is the predominant product using this method (no $\beta$ 1-phosphate was observed using 7 as a substrate) but $\beta$-linked products can be obtained, as evidenced for 10. It is possible that under the acidic reaction conditions interconversion between the $\alpha$ and $\beta$ 1-phosphates occurs, allowing for irreversible cyclic phosphate formation of the $\beta$ anomer, when a suitable C6 electrophile is present,. It remains unconfirmed as to whether this cyclisation could occur during the saponification process (the protected 1-phosphates were not isolated), however no such products were observed for native substrates and the strongly alkaline conditions would likely displace a C6-halide to the parent C6-OH. ${ }^{1} \mathrm{H}$ NMR data for 13 showed a ${ }^{3} J_{\mathrm{H} 1-\mathrm{H} 2}$ coupling constant of $6.9 \mathrm{~Hz}$, which is small for an $\mathrm{H}_{1}-\mathrm{H}_{2}$ axial-axial coupling. DFT calculations were completed for $\alpha$ - and $\beta$ configurations of 13 (Scheme 4b, $\beta$-phosphate shown) which showed a $-24.3 \mathrm{kcal} / \mathrm{mol}$ preference for the $\beta$ and an $\mathrm{H}_{1}-\mathrm{H}_{2}$ dihedral angle of $40.3^{\circ}$. This correlates well to the experimentally observed ${ }^{3} J_{\mathrm{H} 1-\mathrm{H} 2}$ coupling, whereas the dihedral angle obtained for the $\alpha$ linked system was $163.5^{\circ}$ (see SI), which would give rise to a much larger coupling. To the 
best of our knowledge this is the first report of an $O^{1}, O^{6} 7$-membered cyclic phosphate; an $O^{1}, O^{3}$ system was previously reported for D-glucose. ${ }^{[31]}$
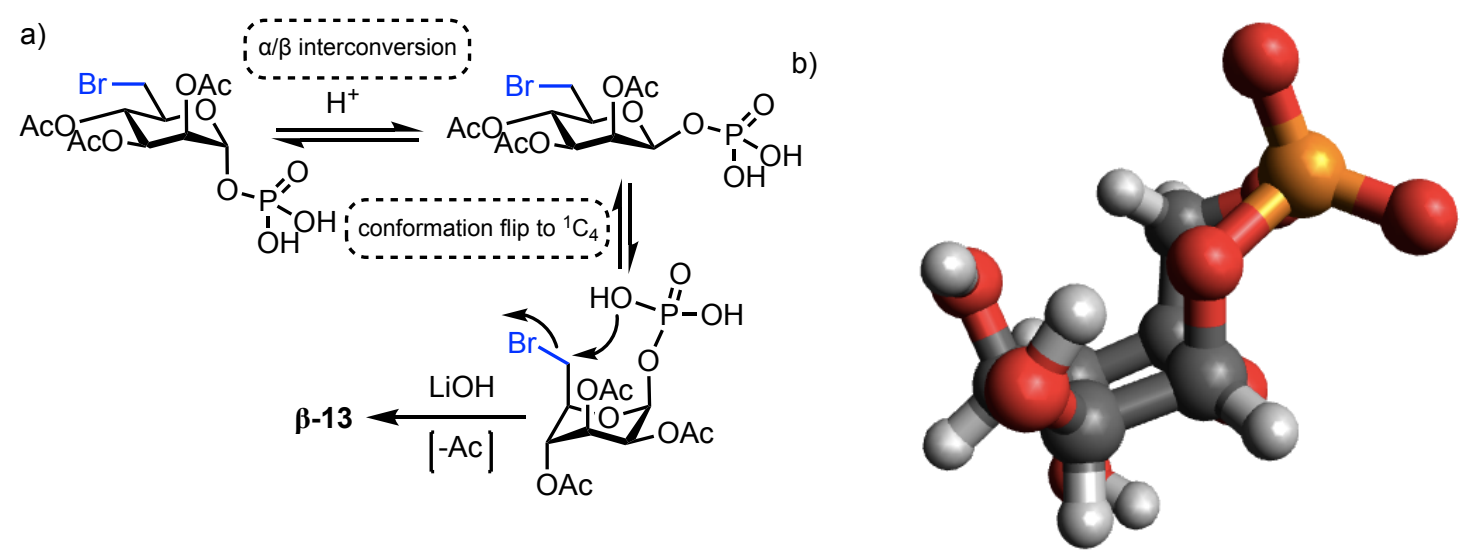

Scheme 4. a) Possible mechanism for the formation of cyclic phosphate $\beta-\mathbf{1 3} b$ ) DFT energy minimised ${ }^{3} \mathrm{C}_{O}$ conformation for $\beta-\mathbf{1 3}$.

To investigate this further (in pursuit of C6-halogenated mannose 1-phosphates), the phosphorylation of the corresponding C6-fluoro derivative $\mathbf{1 4}^{[32]}$ was completed (Table 2, entry 6). As expected with this inferior leaving group, using the same conditions seen for 11, reaction with 14 led to the desired glycosyl 1-phosphate 15 . However, the bicyclic phosphate 13 was still observed, in a three to one (15:13) ratio, as adjudged by ${ }^{1} \mathrm{H}$ NMR (Scheme 3). Intramolecular $\mathrm{S}_{\mathrm{N}} 2$ reactions with aliphatic fluoride are uncommon, but have been described for substrates with reduced conformational flexibility. ${ }^{[33]}$ Lowering the temperature for this reaction to $30{ }^{\circ} \mathrm{C}$ gave the same ratio of products and increasing it to $60{ }^{\circ} \mathrm{C}$ reduced the amount of 15 formed (15:13, 1:1). For comparative purposes, a C6-chloride was synthesised (see Experimental) and, when subject to phosphorylation, was fully converted to 13. These findings suggest that the identity of the halogen is (predictably) key to the rate of competing nucleophilic substitution at C6.

In light of the positive result obtained for accessing 15, phosphorylation of C6deoxy-gem-difluoro D-mannose tetraacetate 16 was attempted (Table 2, entry 7). Pleasingly, 17 was isolated in a moderate yield of $42 \%$. This 6-gem-difluoro substituted material was unreactive to phosphorylation at $40{ }^{\circ} \mathrm{C}$ and conversion was instead completed at $60{ }^{\circ} \mathrm{C}$ and over $6 \mathrm{~h}$. This inevitably induced significant reaction blackening/decomposition, hence the lower yield. It is however encouraging that such an electronically deactivated substrate can be converted to its glycosyl 1-phosphate using this simple process from the tetraacetate.

Investigating other C6-substituted substrates, phosphorylation of 6-deoxy-6-azido Dmannose 18 proceeded smoothly on $100 \mathrm{mg}$ scale and in $65 \%$ yield to deliver 19 (Table 2, entry 8). A C6-thioacetate 20 was accessed from the C6-chloro derivative by nucleophilic substitution using KSAc and enabled an attempted synthesis of 6-thio 1-phosphate 21 (Table 2 , entry 9). The yield for this reaction was lower than that of the native mannose compound $\mathbf{8}$ (43\% compared to 55\%) and disulfide formation for 21 was observed by HRMS and ${ }^{1} \mathrm{H}$ NMR. Purification of this material was further complicated by the free hemi-acetal byproduct also forming a mixed disulfide with 21. Any hemi-acetal by-product was normally removed by anion exchange chromatography, but due to the charged nature of this mixed disulfide it was not possible to isolate pure amounts of $\mathbf{2 1} . \mathrm{H}_{1}$ in the disulfide form of 21 could clearly be observed by ${ }^{1} \mathrm{H}$ NMR, alongside $\mathrm{H}_{1}$ of the mixed disulfide in a ratio of 2.5:1 for 21. Attempts to reduce these disulfides with TCEP (to enable removal of the hemi-acetal) were unsuccessful. 


\section{Conclusions}

An improved access to glycosyl 1-phosphates has been developed, formed using a melt of an acetylated precursor with $\mathrm{H}_{3} \mathrm{PO}_{4}$ under high vacuum. Through preparation of the reaction components in a glove box and reducing the reaction temperature and equivalents of $\mathrm{H}_{3} \mathrm{PO}_{4}$, small scale access to the preparation of native and non-native glycosyl 1-phosphates in good yields and with facile purification is enabled. Additionally, the formation of an unexpected $O^{1}, O^{6}$-cyclic phosphate is reported when good leaving groups $(\mathrm{Br}, \mathrm{Cl})$ at $\mathrm{C} 6$ of the starting material are included. These anomeric phosphates will serve as key tools for the study of enzymes and biochemical processes that utilise glycosyl 1-phosphates, for example, phosphorylases and uridylyltransferases.

\section{Acknowledgments}

Keele University are thanked for PhD studentship funding to LB and ED. The Engineering and Physical Sciences Research Council (EPSRC, EP/P000762/1) and the Biotechnology and Biological Sciences Research Council (BBSRC, BB/L013762/1) are thanked for project grant funding. We also thank the EPSRC (core capability EP/K039466/1) for funding and the EPSRC UK National Mass Spectrometry Facility (NMSF) at Swansea University.

\section{Experimental}

\section{General Experimental}

All reagents and solvents which were available commercially were purchased from Acros, Alfa Aesar, Fisher Scientific, or Sigma Aldrich. All reactions in non-aqueous solvents were conducted in oven dried glassware under a nitrogen atmosphere with a magnetic stirring device. Solvents were purified by passing through activated alumina columns and used directly from a Pure Solv-MD solvent purification system and were transferred under nitrogen. ${ }^{1} \mathrm{H}$ NMR spectra were recorded at 600 or $400 \mathrm{MHz}$ and ${ }^{13} \mathrm{C}$ spectra at $100 \mathrm{MHz}$ respectively using a Bruker AVIII400 spectrometer. ${ }^{1} \mathrm{H}$ NMR signals were assigned with the aid of gDQCOSY. ${ }^{13} \mathrm{C}$ NMR signals were assigned with the aid of gHSQCAD. Coupling constants are reported in Hertz. Chemical shifts $(\delta$, in $\mathrm{ppm})$ are standardised against the deuterated solvent peak. NMR data were analysed using Nucleomatica iNMR or Mestrenova software. ${ }^{1} \mathrm{H}$ NMR splitting patterns were assigned as follows: br s (broad singlet), s (singlet), $\mathrm{d}$ (doublet), app. $\mathrm{t}$ (apparent triplet), $\mathrm{t}$ (triplet), dd (doublet of doublets), ddd (doublet of doublet of doublets), or $\mathrm{m}$ (multiplet and/or multiple resonances). Reactions were followed by thin layer chromatography (TLC) using Merck silica gel 60F254 analytical plates (aluminium support) and were developed using standard visualising agents: short wave UV radiation $(245 \mathrm{~nm})$ and $5 \%$ sulfuric acid in methanol/ $\Delta$. Purification via flash column chromatography was conducted using silica gel $60(0.043-0.063 \mathrm{~mm})$. Optical activities were recorded on automatic polarimeter Rudolph autopol I or Bellingham and Stanley ADP430 (concentration in $\mathrm{g} / 100 \mathrm{~mL}$ ). MS and HRMS (ESI) were obtained on Waters (Xevo, G2-XS TOF) or Waters Micromass LCT spectrometers using a methanol mobile phase. High resolution (ESI) spectra were obtained on a Xevo, G2-XS TOF mass spectrometer. HRMS was obtained using a lock-mass to adjust the calibrated mass. Phosphorylation reactions were prepared in a MBRAUN LABstar glove box.

\section{General procedure anomeric phosphorylation}

The acetylated sugar (1.0 equiv.) was weighed into a pre-dried and weighed Schlenk tube and dried under high vacuum for $1 \mathrm{~h} . \mathrm{H}_{3} \mathrm{PO}_{4}$ (5.0 equiv.) was weighed out inside a glove 
box, transferred to the Schlenk tube and the tube sealed under $\mathrm{N}_{2}$. The tube was placed under $\mathrm{N}_{2}$ on a double manifold and heated under high vacuum at $50{ }^{\circ} \mathrm{C}$ with gentle stirring. In most instances a yellow-gold melt was formed during this time and all reactions were monitored to completion by TLC (petroleum ether/EtOAc, 1/2) for the formation of a baseline $\left(\mathrm{R}_{\mathrm{f}}=0\right)$ spot (1-phosphate) with any hemi-acetal side product generally at $R_{f}=0.5$. The resultant melt was allowed to cool to room temperature then reconstituted in anhydrous THF. This was then added slowly to a stirred solution of $1.0 \mathrm{M} \mathrm{LiOH}$ (concentration $=0.025 \mathrm{M}$ with respect to starting material) at $0{ }^{\circ} \mathrm{C}$ and stirred until saponification was complete by TLC analysis $\left(\mathrm{MeCN} / \mathrm{H}_{2} \mathrm{O} / \mathrm{AcOH}, 3 / 1 / 0.1\right)$, typically $48-72 \mathrm{~h}$. The reaction mixture was filtered through a Whatman GA filter under vacuum and rinsed with deionised water. The resultant filtrate was then neutralised through the addition of Amberlite ${ }^{\mathrm{TM}} 120$ ion-exchange resin $\left(\mathrm{H}^{+}\right.$form $)$to $\mathrm{pH}$ $=7$, filtered and concentrated under reduced pressure. The crude residue was then triturated with $\mathrm{MeOH}$, centrifuged and the supernatant removed The pellet was purified by dissolving in deionised water and passed through a Bio-Scale Mini UNOsphere Q (strong-anion exchange) cartridge [Column Volume $(\mathrm{CV})=5 \mathrm{~mL}$ ], eluting with $3 \mathrm{CV}$ of deionised water followed by $3 \mathrm{CV}$ of $1.0 \mathrm{M} \mathrm{NH}_{4} \mathrm{HCO}_{3}$ solution. Fractions containing the glycosyl-1phosphate were collected and lyophilised repeatedly to remove residual $\mathrm{NH}_{4} \mathrm{HCO}_{3}$ and deliver the target material.

$\alpha$-D-Galactose 1-phosphate bis ammonium salt $\mathbf{2}$

1 (300 mg, $0.77 \mathrm{mmol}$ ) gave 2 (152 mg, $0.52 \mathrm{mmol}, 67 \%)$.

${ }^{1} \mathbf{H}$ NMR $\left(400 \mathrm{MHz}, \mathrm{D}_{2} \mathrm{O}\right) \delta 5.31\left(1 \mathrm{H}, \mathrm{dd}, J_{1, P}=7.3, J_{1,2}=3.6 \mathrm{~Hz}, \mathrm{H}_{1}\right), 4.01(1 \mathrm{H}, \mathrm{dd}, J=7.0$, $\left.4.9 \mathrm{~Hz}, \mathrm{H}_{5}\right), 3.82\left(1 \mathrm{H}, \mathrm{d}, J=2.7 \mathrm{~Hz}, \mathrm{H}_{4}\right), 3.74\left(1 \mathrm{H}, \mathrm{dd}, J=10.2,3.4 \mathrm{~Hz}, \mathrm{H}_{3}\right), 3.57(3 \mathrm{H}, \mathrm{m}$, $\mathrm{H}_{2}, \mathrm{H}_{6 \mathrm{a}}$ and $\left.\mathrm{H}_{6 \mathrm{~b}}\right) ;{ }^{13} \mathrm{C}$ NMR $\left(101 \mathrm{MHz}, \mathrm{D}_{2} \mathrm{O}\right) \delta 94.1\left(\mathrm{C}_{1}\right), 71.0\left(\mathrm{C}_{5}\right), 69.6\left(\mathrm{C}_{3}\right), 69.0\left(\mathrm{C}_{4}\right), 68.7$ $\left(\mathrm{C}_{2}\right), 61.4\left(\mathrm{C}_{6}\right) ;{ }^{31} \mathbf{P}\left\{{ }^{1} \mathbf{H}\right\}$ NMR $\left(162 \mathrm{MHz}, \mathrm{D}_{2} \mathrm{O}\right) \delta$ 2.56. Data matched those previously reported. ${ }^{[28]}$

\section{$\alpha$-D-Glucose 1-phosphate bis sodium salt 4}

3 (200 mg, $0.51 \mathrm{mmol}$ ) gave 4 (54 mg, $0.20 \mathrm{mmol}, 41 \%)$. Following purification as described in the General Experimental, the material was treated with DOWEX-IR-120 ( $\mathrm{Na}^{+}$form) to obtain the bis-sodium salt form for comparison to reported data. ${ }^{1} \mathbf{H}$ NMR $\left(400 \mathrm{MHz}, \mathrm{D}_{2} \mathrm{O}\right)$ $5.44\left(1 \mathrm{H}, \mathrm{dd}, J_{l, P}=7.4, J_{1,2}=3.4 \mathrm{~Hz}, \mathrm{H}_{1}\right), 3.92\left(1 \mathrm{H}, \mathrm{ddd}, J=10.0,5.2,2.1 \mathrm{~Hz}, \mathrm{H}_{5}\right), 3.86(1 \mathrm{H}$, dd, $\left.J=12.2,2.2 \mathrm{~Hz}, \mathrm{H}_{6 \mathrm{~b}}\right), 3.75\left(2 \mathrm{H}, \mathrm{dt}, J=12.3,7.3 \mathrm{~Hz}, \mathrm{H}_{3}, \mathrm{H}_{6 \mathrm{a}}\right), 3.47$ (ddd, $J=9.7,3.3,1.6$ $\left.\mathrm{Hz}, \mathrm{H}_{2}\right), 3.38\left(1 \mathrm{H}\right.$, app. t, $\left.J=9.6 \mathrm{~Hz}, \mathrm{H}_{4}\right) ;{ }^{13} \mathrm{C}$ NMR $\left(101 \mathrm{MHz}, \mathrm{D}_{2} \mathrm{O}\right) \delta 93.5\left(\mathrm{C}_{1}\right), 73.1\left(\mathrm{C}_{3}\right)$, $72.2\left(\mathrm{C}_{5}\right), 71.9\left(\mathrm{C}_{2}\right), 69.7\left(\mathrm{C}_{4}\right), 60.7\left(\mathrm{C}_{6}\right) ;{ }^{31} \mathbf{P}$ NMR $\left(161 \mathrm{MHz}, \mathrm{D}_{2} \mathrm{O}\right) \delta 2.45\left(1 \mathrm{P}, \mathrm{d}, J_{\mathrm{P}, \mathrm{H}}=7.4\right.$ $\mathrm{Hz})$. Data matched those previously reported. ${ }^{[28]}$

\section{2-Deoxy-1,3,4,6-tetra-O-acetyl $\alpha / \beta$-D-glucose 5}

To a stirred solution of 2-deoxy-D-glucose (1.0 g, $6.1 \mathrm{mmol}, 1.0$ equiv.) in anhydrous pyridine $(12 \mathrm{~mL})$ at $0{ }^{\circ} \mathrm{C}$ was added dropwise acetic anhydride $(4.6 \mathrm{~mL}, 48.7 \mathrm{mmol}, 8.0$ equiv.). The reaction mixture was warmed slowly to room temperature then stirred for $15 \mathrm{~h}$, whereby TLC analysis (petroleum ether/EtOAc, 1/1) indicated complete conversion of starting material to a higher $\mathrm{R}_{\mathrm{f}}$ spot. The reaction mixture was poured onto iced water $(100$ $\mathrm{mL})$ and diluted with EtOAc $(75 \mathrm{~mL})$. The organic layer was washed successively with $1.0 \mathrm{M}$ $\mathrm{HCl}$, saturated aqueous $\mathrm{NaHCO}_{3}$ solution, water and brine $(75 \mathrm{~mL}$ each). The aqueous layer was re-extracted with EtOAc $(100 \mathrm{~mL})$ and the combined organic layers dried $\left(\mathrm{MgSO}_{4}\right)$, filtered and concentrated under reduced pressure. The resultant oil was co-evaporated with toluene $(3 \times 20 \mathrm{~mL})$ to afford 5 as a white solid $(1.84 \mathrm{~g}, 5.54 \mathrm{mmol}, 91 \%) . \mathrm{R}_{\mathrm{f}} 0.74$ (petroleum ether/EtOAc, $1 / 1) ;{ }^{1} \mathbf{H}$ NMR $\left(400 \mathrm{MHz}, \mathrm{CDCl}_{3}\right) 5: 1 \beta / \alpha, \delta(\beta$-anomer) $5.77(1 \mathrm{H}$, dd, $\left.J=10.0,2.3 \mathrm{~Hz}, \mathrm{H}_{1}\right), 5.06-4.97\left(2 \mathrm{H}, \mathrm{m}, \mathrm{H}_{3}, \mathrm{H}_{4}\right), 4.29\left(1 \mathrm{H}, \mathrm{dd}, J=12.4,4.7 \mathrm{~Hz}, \mathrm{H}_{6 \mathrm{a}}\right)$, 
$4.06\left(1 \mathrm{H}, \mathrm{dd}, J=12.4,2.2 \mathrm{~Hz}, \mathrm{H}_{6 \mathrm{~b}}\right), 3.72\left(1 \mathrm{H}, \mathrm{ddd}, J=9.3,4.6,2.2 \mathrm{~Hz}, \mathrm{H}_{5}\right), 2.32(1 \mathrm{H}, \mathrm{ddd}, J$ $\left.=12.6,4.9,2.3 \mathrm{~Hz}, \mathrm{H}_{2}\right), 2.09\left(3 \mathrm{H}, \mathrm{s}, \mathrm{C}(\mathrm{O}) \mathrm{CH}_{3}\right), 2.06\left(3 \mathrm{H}, \mathrm{s}, \mathrm{C}(\mathrm{O}) \mathrm{CH}_{3}\right), 2.02(3 \mathrm{H}, \mathrm{s}$, $\left.\mathrm{C}(\mathrm{O}) \mathrm{CH}_{3}\right), 2.01\left(3 \mathrm{H}, \mathrm{s}, \mathrm{C}(\mathrm{O}) \mathrm{CH}_{3}\right) ;{ }^{13} \mathbf{C}$ NMR $\left(100 \mathrm{MHz}, \mathrm{CDCl}_{3}\right) \delta(\beta$-anomer $) 170.8(\mathrm{C}=\mathrm{O})$, $170.2(\mathrm{C}=\mathrm{O}), 169.8(\mathrm{C}=\mathrm{O}), 168.9(\mathrm{C}=\mathrm{O}), 91.2\left(\mathrm{C}_{1}\right), 72.9\left(\mathrm{C}_{5}\right), 70.2\left(\mathrm{C}_{4}\right), 68.4\left(\mathrm{C}_{3}\right), 62.1\left(\mathrm{C}_{6}\right)$, $34.8\left(\mathrm{C}_{2}\right), 21.0\left(\mathrm{C}(\mathrm{O}) \mathrm{CH}_{3}\right), 20.9\left(\mathrm{C}(\mathrm{O}) \mathrm{CH}_{3}\right), 20.9\left(\mathrm{C}(\mathrm{O}) \mathrm{CH}_{3}\right), 20.8\left(\mathrm{C}(\mathrm{O}) \mathrm{CH}_{3}\right)$; HRMS m/z $\left(\mathrm{ESI}^{+}\right)$Found: $(\mathrm{M}+\mathrm{Na})^{+} 355.1010, \mathrm{C}_{14} \mathrm{H}_{20} \mathrm{O}_{9} \mathrm{Na}$ requires 355.1005. Data matched those previously reported. ${ }^{[34]}$

\section{$\alpha$-D-Mannose 1-phosphate bis sodium salt $\mathbf{8}$}

7 (250 mg, $0.64 \mathrm{mmol})$ gave 8 (91 $\mathrm{mg}, 0.35 \mathrm{mmol}, 55 \%)$. Reaction temperature was $45{ }^{\circ} \mathrm{C}$, following purification as described in the General Experimental, the material was treated with DOWEX-IR-120 ( $\mathrm{Na}^{+}$form) to obtain the bis-sodium salt form for comparison to reported data. $\mathrm{R}_{\mathrm{f}} 0.40$ (acetonitrile/water/ $\left.\mathrm{NH}_{4} \mathrm{OH}, 2 / 1 / 0.1\right) ;[\alpha]_{D}^{26}+22.2\left(c=0.45, \mathrm{H}_{2} \mathrm{O}\right) ;{ }^{1} \mathbf{H}$ NMR $(400$ $\left.\mathrm{MHz}, \mathrm{D}_{2} \mathrm{O}\right) \delta 5.23\left(1 \mathrm{H}, \mathrm{d}, J_{1, \mathrm{P}}=8.5 \mathrm{~Hz}, \mathrm{H}_{1}\right), 3.87-3.74\left(4 \mathrm{H}, \mathrm{m}, \mathrm{H}_{2}, \mathrm{H}_{3}, \mathrm{H}_{5}, \mathrm{H}_{6 \mathrm{a}}\right), 3.66-3.59$ $\left(1 \mathrm{H}, \mathrm{m}, \mathrm{H}_{6 \mathrm{~b}}\right) 3.50\left(1 \mathrm{H}\right.$, app. $\left.\mathrm{t}, J=9.6 \mathrm{~Hz}, \mathrm{H}_{4}\right) ;{ }^{13} \mathbf{C} \mathbf{N M R}\left(100 \mathrm{MHz}, \mathrm{D}_{2} \mathrm{O}\right) \delta 95.0\left(\mathrm{C}_{1}\right), 73.0$, 71.2, 70.2, $67.2\left(\mathrm{C}_{4}\right), 61.3\left(\mathrm{C}_{6}\right) ;{ }^{31} \mathbf{P}$ NMR $\left(161 \mathrm{MHz}, \mathrm{D}_{2} \mathrm{O}\right) \delta 2.00\left(1 \mathrm{P}, \mathrm{d}, J_{\mathrm{P}, \mathrm{H}}=8.5 \mathrm{~Hz}\right)$; HRMS $m / z$ (ES-) Found: $(\mathrm{M}-\mathrm{H})^{-} 259.0224, \mathrm{C}_{6} \mathrm{H}_{11} \mathrm{O}_{9}$ requires 259.0231. Data matched those previously reported. ${ }^{[35]}$

\section{1,2,3,4,6-Penta-O-acetyl-5-C-Methyl- $\alpha / \beta$-D-mannose 9}

Acetylation using $\mathrm{Ac}_{2} \mathrm{O}$ /pyridine

5 - $C$-methyl- $\alpha / \beta$-D-mannose ${ }^{[29]}(1.1 \mathrm{~g}, 5.8 \mathrm{mmol}, 1.0$ equiv. $)$ was stirred in acetic anhydride $(20 \mathrm{~mL})$ and pyridine $(40 \mathrm{~mL})$ for 18 hours at room temperature under a nitrogen atmosphere. The reaction mixture was concentrated in vacuo and co-evaporated with toluene $(3 \mathrm{x} 20 \mathrm{~mL})$. TLC $\left(3 \% \mathrm{MeOH}\right.$ in DCM) showed four spots $\left(\mathrm{R}_{\mathrm{f}}\right.$ 0.39-0.75) corresponding to the diastereomeric pyranoside and furanoside forms. This residue was purified by silica gel column chromatography to afford 9 as an inseparable mixture of pyranosides and $\alpha$ furanoside ( $>93 \%$ pyranoside by ${ }^{1} \mathrm{H}$ NMR integration and a 58:35 $\beta: \alpha$ pyranoside ratio) as an off-white solid (688 mg, $1.73 \mathrm{mmol}, 67 \%$ ). The $\beta$-furanoside was separable as a yellow, flaky solid (91 mg, $0.23 \mathrm{mmol}, 4 \%) .{ }^{1} \mathbf{H}$ NMR $\left(400 \mathrm{MHz}, \mathrm{CDCl}_{3}\right) \delta \alpha$-pyranoside $6.11(1 \mathrm{H}, \mathrm{d}, J=$ $\left.2.3 \mathrm{~Hz}, \mathrm{H}_{1}\right), 5.58\left(1 \mathrm{H}, \mathrm{d}, J=10.5 \mathrm{~Hz}, \mathrm{H}_{4}\right), 5.25\left(2 \mathrm{H}, \mathrm{dd}, J=10.2,3.3 \mathrm{~Hz}, \mathrm{H}_{2}, \mathrm{H}_{3}\right), 4.11-3.95$ $\left(2 \mathrm{H}, \mathrm{m}, \mathrm{H}_{6 \mathrm{a}+\mathrm{b}}\right), 2.02\left(3 \mathrm{H}, \mathrm{s},\left(\mathrm{C}(\mathrm{O}) \mathrm{CH}_{3}\right), 2.06\left(3 \mathrm{H}, \mathrm{s},\left(\mathrm{C}(\mathrm{O}) \mathrm{CH}_{3}\right), 2.12\left(3 \mathrm{H}, \mathrm{s},\left(\mathrm{C}(\mathrm{O}) \mathrm{CH}_{3}\right)\right.\right.\right.$, $2.14\left(3 \mathrm{H}, \mathrm{s},\left(\mathrm{C}(\mathrm{O}) \mathrm{CH}_{3}\right), 2.17\left(3 \mathrm{H}, \mathrm{s},\left(\mathrm{C}(\mathrm{O}) \mathrm{CH}_{3}\right), 1.39\left(3 \mathrm{H}, \mathrm{s}, \mathrm{CH}_{3}\right) ; \beta\right.\right.$-pyranoside $6.06(1 \mathrm{H}$, d, $\left.J=1.4 \mathrm{~Hz}, \mathrm{H}_{1}\right), 5.48\left(1 \mathrm{H}, \mathrm{d}, J=10.4 \mathrm{~Hz}, \mathrm{H}_{4}\right), 5.25\left(2 \mathrm{H}, \mathrm{dd}, J=10.2,3.3 \mathrm{~Hz}, \mathrm{H}_{2}, \mathrm{H}_{3}\right)$, 4.11-3.95 (2H, m, $\left.\mathrm{H}_{6 \mathrm{a}+\mathrm{b}}\right) 2.01\left(3 \mathrm{H}, \mathrm{s},\left(\mathrm{C}(\mathrm{O}) \mathrm{CH}_{3}\right), 2.05\left(3 \mathrm{H}, \mathrm{s},\left(\mathrm{C}(\mathrm{O}) \mathrm{CH}_{3}\right), 2.10(3 \mathrm{H}, \mathrm{s}\right.\right.$, $\left(\mathrm{C}(\mathrm{O}) \mathrm{CH}_{3}\right), 2.12\left(3 \mathrm{H}, \mathrm{s},\left(\mathrm{C}(\mathrm{O}) \mathrm{CH}_{3}\right), 2.21\left(3 \mathrm{H}, \mathrm{s},\left(\mathrm{C}(\mathrm{O}) \mathrm{CH}_{3}\right), 1.40\left(3 \mathrm{H}, \mathrm{s}, \mathrm{CH}_{3}\right) ; \alpha\right.\right.$-furanoside $5.41\left(1 \mathrm{H}, \mathrm{s}, \mathrm{H}_{1}\right), 5.21\left(1 \mathrm{H}, \mathrm{m}, \mathrm{H}_{2}\right) 4.99\left(1 \mathrm{H}, \mathrm{dd}, J=5.6,1.8 \mathrm{~Hz}, \mathrm{H}_{3}\right), 4.91(1 \mathrm{H}, \mathrm{d}, J=1.8 \mathrm{~Hz}$, $\left.\mathrm{H}_{4}\right), 4.35,3.56\left(2 \mathrm{H}, \mathrm{q}, J=7.7 \mathrm{~Hz}, \mathrm{H}_{6 \mathrm{a}+6 \mathrm{~b}}\right), 2.19\left(3 \mathrm{H}, \mathrm{s},\left(\mathrm{C}(\mathrm{O}) \mathrm{CH}_{3}\right), 2.15\left(3 \mathrm{H}, \mathrm{s},\left(\mathrm{C}(\mathrm{O}) \mathrm{CH}_{3}\right)\right.\right.$, $2.07\left(3 \mathrm{H}, \mathrm{s},\left(\mathrm{C}(\mathrm{O}) \mathrm{CH}_{3}\right), 1.98\left(3 \mathrm{H}, \mathrm{s},\left(\mathrm{C}(\mathrm{O}) \mathrm{CH}_{3}\right), 1.34\left(3 \mathrm{H}, \mathrm{s}, \mathrm{CH}_{3}\right), \beta\right.\right.$-furanoside $6.25(1 \mathrm{H}, \mathrm{d}$, $\left.J=3.2 \mathrm{~Hz}, \mathrm{H}_{1}\right), 5.71-5.68\left(1 \mathrm{H}, \mathrm{m}, \mathrm{H}_{3}\right), 5.41\left(1 \mathrm{H}, \mathrm{dd}, J=5.1,3.2 \mathrm{~Hz}, \mathrm{H}_{4}\right), 4.27(1 \mathrm{H}, \mathrm{d}, J=$ $\left.4.3 \mathrm{~Hz}, \mathrm{H}_{2}\right), 4.15-4.14\left(2 \mathrm{H}, \mathrm{m}, \mathrm{H}_{6 \mathrm{a}+\mathrm{b}}\right), 2.11\left(3 \mathrm{H}, \mathrm{s},\left(\mathrm{C}(\mathrm{O}) \mathrm{CH}_{3}\right)\right), 2.10-2.10(6 \mathrm{H}, \mathrm{m}$, $\left(\mathrm{C}(\mathrm{O}) \mathrm{CH}_{3}\right), 2.07\left(3 \mathrm{H}, \mathrm{s},\left(\mathrm{C}(\mathrm{O}) \mathrm{CH}_{3}\right), 1.29\left(3 \mathrm{H}, \mathrm{s}, \mathrm{CH}_{3}\right) ;{ }^{13} \mathbf{C} \mathbf{N M R}\left(101 \mathrm{MHz}, \mathrm{CDCl}_{3}\right)\right.$ For pyranoses $\delta 170.6,170.5,170.2,170.0,169.9,169.7,169.5,169.4,168.6,168.4,91.1\left(\mathrm{C}_{1 \beta}\right)$, $86.8\left(\mathrm{C}_{1 \alpha}\right), 78.2\left(\mathrm{C}_{5 \beta}\right), 76.1\left(\mathrm{C}_{5 \alpha}\right), 68.4,68.3,67.3\left(\mathrm{C}_{6 \alpha}\right), 67.2\left(\mathrm{C}_{6 \beta}\right), 66.5,21.2,20.8,20.8$, 20.8, 20.7, 20.6, $19.3\left(\mathrm{CH}_{3-\beta}\right), 15.0\left(\mathrm{CH}_{3-\alpha}\right)$; HRMS $\mathrm{m} / z\left(\mathrm{ES}^{+}\right)$Found: $(\mathrm{M}+\mathrm{Na})^{+}$427.1215, $\mathrm{C}_{17} \mathrm{H}_{24} \mathrm{O}_{11} \mathrm{Na}$ requires $(\mathrm{M}+\mathrm{Na})^{+} 427.1217$.

Acetylation using $\mathrm{Ac}_{2} \mathrm{O}_{2} \mathrm{H}_{2} \mathrm{SO}_{4}$

To a stirred mixture of $\mathrm{Ac}_{2} \mathrm{O}(850 \mu \mathrm{L}, 9.0 \mathrm{mmol}, 10.3$ equiv. $)$ and 5 - $C$-methyl- $\alpha / \beta$-Dmannose ${ }^{[29]}\left(170 \mathrm{mg}, 0.87 \mathrm{mmol}, 1.0\right.$ equiv.), $\mathrm{H}_{2} \mathrm{SO}_{4}$ (1 drop) was added at $0{ }^{\circ} \mathrm{C}$, under an atmosphere of $\mathrm{N}_{2}$. The solution was stirred for $10 \mathrm{~min}$ at $0{ }^{\circ} \mathrm{C}$ and then allowed to warm to 
room temperature and stirred for a further $45 \mathrm{~min}$. The mixture was then diluted with icewater $(30 \mathrm{~mL})$, and the organic phase extracted with EtOAc $(50 \mathrm{~mL})$. The extract was washed with water $(3 \times 20 \mathrm{~mL})$, sat. aq. $\mathrm{NaHCO}_{3}$ solution $(30 \mathrm{~mL})$, dried over $\mathrm{MgSO}_{4}$, filtered, and the solvent evaporated to dryness to yield $\mathbf{9}$ as diastereomeric pyranoside and furanoside forms as a pale-yellow viscous oil (9.9 g, $0.69 \mathrm{mmol}, 79 \%) .{ }^{1} \mathrm{H}$ NMR integration showed $>83 \%$ pyranoside and a 43:40 $\beta: \alpha$ pyranoside ratio. ${ }^{13}$ C-GATED $\left(101 \mathrm{MHz} ; \mathrm{CDCl}_{3}\right): 91.1$ $\left({ }^{1} J_{C l-H 1}=176 \mathrm{~Hz}, \mathrm{C}_{1 \alpha}\right), 86.8\left({ }^{1} J_{C 1^{\prime}-H 1}{ }^{\prime}=164 \mathrm{~Hz}, \mathrm{C}_{1 \beta}\right)$. Other analytical data matched those presented above and as previously reported. ${ }^{[29]}$

\section{5-C-Methyl- $\alpha / \beta$-D-mannose 1-phosphate bis ammonium salt $\mathbf{1 0}$}

From 9 (250 mg, $0.69 \mathrm{mmol}$ ) gave 10 (141 mg, $0.46 \mathrm{mmol}, 66 \%)$.

${ }^{1} \mathbf{H}$ NMR $\left(400 \mathrm{MHz}, \mathrm{D}_{2} \mathrm{O}\right) \delta \alpha$-anomer $5.27\left(1 \mathrm{H}, \mathrm{dd}, J_{l, P}=9.1, J_{l, 2}=2.2 \mathrm{~Hz}, \mathrm{H}_{1}\right), 4.09(1 \mathrm{H}$, $\left.\mathrm{dd}, J=10.2,3.3 \mathrm{~Hz}, \mathrm{H}_{3}\right), 3.93-3.91\left(1 \mathrm{H}, \mathrm{m}, \mathrm{H}_{2}\right), 3.78\left(1 \mathrm{H}, \mathrm{d}, J=10.2 \mathrm{~Hz}, \mathrm{H}_{4}\right), 3.39(2 \mathrm{H}, \mathrm{q}, J$ $\left.=11.9 \mathrm{~Hz}, \mathrm{H}_{6 \mathrm{a}+\mathrm{b}}\right) ; \beta$-anomer $\delta 5.19\left(1 \mathrm{H}, \mathrm{dd}, J=8.6,1.1 \mathrm{~Hz}, \mathrm{H}_{1}\right), 3.96\left(1 \mathrm{H}, \mathrm{d}, J=2.8 \mathrm{~Hz}, \mathrm{H}_{2}\right)$, $3.84\left(1 \mathrm{H}, \mathrm{dd}, J=10.2,3.4 \mathrm{~Hz}, \mathrm{H}_{3}\right), 3.64\left(1 \mathrm{H}, \mathrm{d}, J=10.2 \mathrm{~Hz}, \mathrm{H}_{4}\right), 3.54-3.45\left(2 \mathrm{H}, \mathrm{m}, \mathrm{H}_{6 \mathrm{a}+\mathrm{b}}\right)$; ${ }^{13} \mathrm{C}$ NMR $\left(101 \mathrm{MHz}, \mathrm{D}_{2} \mathrm{O}\right) \delta \alpha$-anomer $95.8(\mathrm{~d}, J=3.9 \mathrm{~Hz}), 79.8,68.0,67.2,66.7,66.0$, $18.0 ;{ }^{31} \mathbf{P}\left\{{ }^{1} \mathbf{H}\right\}$ NMR $\left(162 \mathrm{MHz}, \mathrm{D}_{2} \mathrm{O}\right) \delta 1.88,2.31$; HRMS $m / z\left(\mathrm{ES}^{-}\right)$Found: $(\mathrm{M}-\mathrm{H})^{-}$ 273.0385, $\mathrm{C}_{7} \mathrm{H}_{14} \mathrm{O}_{9} \mathrm{P}$ requires 273.0381.

\section{1,2,3,4-Tetra-O-acetyl-6-bromo-6-deoxy- $\beta$-D-mannose 11}

Triphenylphosphine (2.25 g, $8.60 \mathrm{mmol}, 2.0$ equiv.) was added to a stirred solution of 1,2,3,4-tetra-O-acetyl- $\beta$-D-mannose ${ }^{[30]}(1.50 \mathrm{~g}, 4.30 \mathrm{mmol}, 1.0$ equiv. $)$ in anhydrous pyridine $(43 \mathrm{~mL})$ at room temperature under a $\mathrm{N}_{2}$ atmosphere. The reaction mixture was cooled to 0 ${ }^{\circ} \mathrm{C}$ and carbon tetrabromide ( $3.08 \mathrm{~g}, 9.30 \mathrm{mmol}, 2.2$ equiv.) was added portion wise over 5 min. After $16 \mathrm{~h}$ at $\mathrm{rt}$, TLC analysis (hexane/EtOAc, 3/1) showed complete conversion of starting material to a higher $\mathrm{R}_{\mathrm{f}}$ value spot. The solvent was removed under reduced pressure and the residue re-suspended in EtOAc $(30 \mathrm{~mL})$, washed with $\mathrm{H}_{2} \mathrm{O}(20 \mathrm{~mL})$ and brine $(20$ $\mathrm{mL})$. The organic phase was dried over $\mathrm{MgSO}_{4}$, filtered and concentrated under vacuum. Purification using silica gel column chromatography (hexane/EtOAc, 8:2) furnished 11 (1.52 $\mathrm{g}, 3.70 \mathrm{mmol}, 86 \%$ ) as a pale yellow solid. $\mathrm{R}_{\mathrm{f}} 0.77$ (petroleum ether/EtOAc, $1 / 1$ ); $[\alpha]_{D}^{26}-14.6$ $\left(c=0.55, \mathrm{CHCl}_{3}\right) ;{ }^{1} \mathbf{H}$ NMR $\left(400 \mathrm{MHz}, \mathrm{CDCl}_{3}\right) \delta 5.89\left(1 \mathrm{H}, \mathrm{d}, J=1.1 \mathrm{~Hz}, \mathrm{H}_{1}\right), 5.47(1 \mathrm{H}, \mathrm{dd}$, $\left.J=3.2,1.2 \mathrm{~Hz}, \mathrm{H}_{2}\right), 5.27\left(1 \mathrm{H}\right.$, appt, $\left.J=9.7 \mathrm{~Hz}, \mathrm{H}_{4}\right), 5.13\left(1 \mathrm{H}, \mathrm{ddd}, J=9.8,3.2,1.2 \mathrm{~Hz}, \mathrm{H}_{3}\right)$, $3.82\left(1 \mathrm{H}, \mathrm{ddd}, J=9.4,6.2,3.0 \mathrm{~Hz}, \mathrm{H}_{5}\right), 3.50\left(1 \mathrm{H}, \mathrm{dd}, J=11.4,3.0 \mathrm{~Hz}, \mathrm{H}_{6 \mathrm{a}}\right), 3.42(1 \mathrm{H}, \mathrm{dd}, J$ $\left.=11.5,6.4 \mathrm{~Hz}, \mathrm{H}_{6 \mathrm{~b}}\right), 2.20\left(3 \mathrm{H}, \mathrm{s}, \mathrm{C}(\mathrm{O}) \mathrm{OCH}_{3}\right), 2.10\left(3 \mathrm{H}, \mathrm{s}, \mathrm{C}(\mathrm{O}) \mathrm{OCH}_{3}\right), 2.07(3 \mathrm{H}, \mathrm{s}$, $\left.\mathrm{C}(\mathrm{O}) \mathrm{OCH}_{3}\right), 1.99\left(3 \mathrm{H}, \mathrm{s}, \mathrm{C}(\mathrm{O}) \mathrm{OCH}_{3}\right) ;{ }^{13} \mathbf{C} \mathbf{~ N M R}\left(100 \mathrm{MHz}, \mathrm{CDCl}_{3}\right) \delta 170.3(\mathrm{C}=\mathrm{O}), 169.9$ $(\mathrm{C}=\mathrm{O}), 169.7(\mathrm{C}=\mathrm{O}), 168.5(\mathrm{C}=\mathrm{O}), 90.4\left(\mathrm{C}_{1}\right), 74.5\left(\mathrm{C}_{5}\right), 70.6\left(\mathrm{C}_{3}\right), 68.3\left(\mathrm{C}_{2}\right), 68.1\left(\mathrm{C}_{4}\right), 30.3$ $\left(\mathrm{C}_{6}\right), 20.9\left(2 \mathrm{C}, \mathrm{C}(\mathrm{O}) \mathrm{CH}_{3}\right), 20.8\left(2 \mathrm{C}, \mathrm{C}(\mathrm{O}) \mathrm{CH}_{3}\right), 20.6\left(2 \mathrm{C}, \mathrm{C}(\mathrm{O}) \mathrm{CH}_{3}\right) ; \mathbf{H R M S ~ m} / z\left(\mathrm{ESI}^{+}\right)$ Found: $(\mathrm{M}+\mathrm{Na})^{+} 433.0115, \mathrm{C}_{14} \mathrm{H}_{19} \mathrm{BrO}{ }_{9} \mathrm{Na}$ requires $(\mathrm{M}+\mathrm{Na})^{+} 433.0110$. Previously reported data were for the $\alpha$-anomer. ${ }^{[36]}$

\section{1,2,3,4-Tetra-O-acetyl-6-chloro-6-deoxy- $\beta$-D-mannose}

To a stirred solution of 1,2,3,4-tetra-O-acetyl- $\beta$-D-mannose $e^{[30]}(500 \mathrm{mg}, 1.44 \mathrm{mmol}, 1.0$ equiv.) and triphenylphosphine (640 mg, $2.45 \mathrm{mmol}, 1.7$ equiv.) in anhydrous dichloromethane $(14 \mathrm{~mL})$ under inert $\mathrm{N}_{2}$ atmosphere at $0{ }^{\circ} \mathrm{C}$ was added dropwise carbon tetrachloride $\left(0.24 \mathrm{~mL}, 2.45 \mathrm{mmol}, 1.7\right.$ equiv.). The reaction mixture was stirred at $0{ }^{\circ} \mathrm{C}$ for $30 \mathrm{~min}$ then warmed gradually to room temperature. The reaction mixture was then heated to $40{ }^{\circ} \mathrm{C}$ for $6 \mathrm{~h}$, whereby TLC analysis (petroleum ether/EtOAc, 1/1) showed conversion of starting material to a higher $\mathrm{R}_{\mathrm{f}}$ value spot. The reaction mixture was poured onto distilled water $(30 \mathrm{~mL})$ and diluted with dichloromethane $(35 \mathrm{~mL})$. The organic layer was washed with distilled water $(2 \times 30 \mathrm{~mL})$ and brine $(30 \mathrm{~mL})$ then the aqueous layer was re-extracted 
with dichloromethane $(35 \mathrm{~mL})$. The combined organic layers were dried $\left(\mathrm{MgSO}_{4}\right)$, filtered and concentrated under reduced pressure. Purification by silica gel column chromatography, eluting with petroleum ether/EtOAc $(4 / 1,3 / 1,1 / 1)$ to afford the title compound (320 $\mathrm{mg}, 0.82$ mmol, 61\%) as a white foam. $\mathrm{R}_{\mathrm{f}} 0.78$ (petroleum ether/EtOAc, $\left.1 / 1\right) ;[\alpha]_{D}^{26}-13.3$ (c 0.75 , $\left.\mathrm{CHCl}_{3}\right) ;{ }^{1} \mathbf{H}$ NMR $\left(400 \mathrm{MHz}, \mathrm{CDCl}_{3}\right) \delta 5.87\left(1 \mathrm{H}, \mathrm{d}, J=0.7 \mathrm{~Hz}, \mathrm{H}_{1}\right), 5.44(1 \mathrm{H}, \mathrm{d}, J=2.3 \mathrm{~Hz}$, $\left.\mathrm{H}_{2}\right), 5.26\left(1 \mathrm{H}\right.$, app. t, $\left.J=9.7 \mathrm{~Hz}, \mathrm{H}_{4}\right), 5.12\left(1 \mathrm{H}, \mathrm{dd}, J=9.9,3.2 \mathrm{~Hz}, \mathrm{H}_{3}\right), 3.84-3.78(1 \mathrm{H}, \mathrm{m}$, $\left.\mathrm{H}_{5}\right), 3.64\left(1 \mathrm{H}, \mathrm{dd}, J=12.2,2.9 \mathrm{~Hz}, \mathrm{H}_{6 \mathrm{~b}}\right), 3.57\left(1 \mathrm{H}, \mathrm{dd}, J=12.2,5.8 \mathrm{~Hz}, \mathrm{H}_{6 \mathrm{a}}\right), 2.16(3 \mathrm{H}, \mathrm{s}$, $\left.\mathrm{C}(\mathrm{O}) \mathrm{OCH}_{3}\right), 2.07\left(3 \mathrm{H}, \mathrm{s}, \mathrm{C}(\mathrm{O}) \mathrm{OCH}_{3}\right), 2.04\left(3 \mathrm{H}, \mathrm{s}, \mathrm{C}(\mathrm{O}) \mathrm{OCH}_{3}\right), 1.96\left(3 \mathrm{H}, \mathrm{s}, \mathrm{C}(\mathrm{O}) \mathrm{OCH}_{3}\right) ;{ }^{13} \mathbf{C}$ NMR $\left(100 \mathrm{MHz}, \mathrm{CDCl}_{3}\right) \delta 170.2(\mathrm{C}=\mathrm{O}), 169.8(\mathrm{C}=\mathrm{O}), 169.6(\mathrm{C}=\mathrm{O}), 168.4(\mathrm{C}=\mathrm{O}), 90.3\left(\mathrm{C}_{1}\right)$, $74.7\left(\mathrm{C}_{5}\right), 70.5\left(\mathrm{C}_{3}\right), 68.2\left(\mathrm{C}_{2}\right), 66.9\left(\mathrm{C}_{4}\right), 42.9\left(\mathrm{C}_{6}\right), 20.8\left(\mathrm{C}(\mathrm{O}) \mathrm{CH}_{3}\right), 20.7\left(2 \mathrm{C} 2 \times \mathrm{C}(\mathrm{O}) \mathrm{CH}_{3}\right)$, $20.6\left(\mathrm{C}(\mathrm{O}) \mathrm{CH}_{3}\right)$; HRMS $\mathrm{m} / z\left(\mathrm{ESI}^{+}\right)$Found: $(\mathrm{M}+\mathrm{Na})^{+} 389.0633, \mathrm{C}_{14} \mathrm{H}_{19} \mathrm{ClO}_{9} \mathrm{Na}$ requires $[\mathrm{M}+\mathrm{Na}]^{+} 389.0616$.

$O^{1}, O^{6}$-Hydroxyhosphoryl-D-mannopyranose ammonium salt $\mathbf{1 3}$

Synthesised from 11

11 (400 mg, $0.97 \mathrm{mmol}$ ) gave 13 (200 mg, $0.82 \mathrm{mmol}, 85 \%)$.

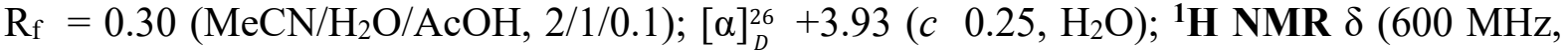
$\left.\mathrm{D}_{2} \mathrm{O}\right) \delta 5.20\left(1 \mathrm{H}\right.$, app. t, $\left.J_{1, P \& 1,2}=6.9 \mathrm{~Hz}, \mathrm{H}_{1}\right), 4.29\left(1 \mathrm{H}\right.$, app. t, $\left.J=2.8 \mathrm{~Hz}, \mathrm{H}_{5}\right), 4.20(1 \mathrm{H}$, dd, $\left.J=6.0,2.8 \mathrm{~Hz}, \mathrm{H}_{4}\right), 4.14\left(1 \mathrm{H}\right.$, br. d, $\left.J=6.5 \mathrm{~Hz}, \mathrm{H}_{3}\right), 4.10\left(1 \mathrm{H}, \mathrm{d}, J=10.9 \mathrm{~Hz}, \mathrm{H}_{6 \mathrm{a}}\right), 3.96(1 \mathrm{H}$, dd, $\left.J=10.8,3.0 \mathrm{~Hz}, \mathrm{H}_{6 \mathrm{~b}}\right), 3.76\left(1 \mathrm{H}, \mathrm{dd}, J=6.6,1.2 \mathrm{~Hz}, \mathrm{H}_{2}\right) ;{ }^{13} \mathbf{C}$ NMR $\left(125 \mathrm{MHz}, \mathrm{D}_{2} \mathrm{O}\right) \delta$ $96.5\left(\mathrm{C} 1, \mathrm{~d},{ }^{3} J_{C, P}=3.2 \mathrm{~Hz}\right), 76.9(\mathrm{C} 3), 75.3(\mathrm{C} 5), 70.3\left(\mathrm{C} 2, \mathrm{~d},{ }^{4} J_{C, P}=4.4 \mathrm{~Hz}\right), 70.0(\mathrm{C} 4), 68.9$ (C6); ${ }^{31} \mathbf{P}\left\{{ }^{1} \mathbf{H}\right\}$ NMR $\left(162 \mathrm{MHz}, \mathrm{D}_{2} \mathrm{O}\right) \delta 2.41$; HRMS $m / z$ (ESI $\left.{ }^{-}\right)$Found: (M-H) 241.0116 , $\mathrm{C}_{6} \mathrm{H}_{10} \mathrm{O}_{8} \mathrm{P}$ requires $(\mathrm{M}-\mathrm{H})^{-} 241.0119$.

Synthesised from 6-chloro-6-deoxy-1,2,3,4-tetra- $O$-acetyl- $\beta$-D-mannose

6-chloro-6-deoxy-1,2,3,4-tetra- $\boldsymbol{O}$-acetyl- $\boldsymbol{\beta}$-D-mannose $(150 \mathrm{mg}, 0.41 \mathrm{mmol})$ gave 13 (15 $\mathrm{mg}, 0.07 \mathrm{mmol}, 17 \%)$. Analytical data matched those above.

\section{1,2,3,4-Tetra-O-acetyl-6-deoxy-6-fluoro- $\beta$-D-mannopyranose 14}

Synthesised from 1,2,3,4-tetra- $O$-acetyl- $\beta$-D-mannose,,${ }^{[30]}$ according to literature procedures. ${ }^{[32]}$

\section{6-Deoxy-6-fluoro- $\alpha$-D-mannose 1-phosphate bis ammonium salt $\mathbf{1 5}$}

14 (54 mg, $0.15 \mathrm{mmol})$ gave 15 (40 mg, $0.15 \mathrm{mmol}, 72 \%, 15: 13=1.0: 0.3)$.

Data for 15: ${ }^{1} \mathbf{H}\left\{{ }^{19} \mathbf{F}\right\}$ NMR $\left(600 \mathrm{MHz}, \mathrm{D}_{2} \mathrm{O}\right) \delta 5.26\left(\mathrm{dd}, J_{1, P}=8.5, J_{1,2}=1.0 \mathrm{~Hz}, \mathrm{H}_{1}\right), 4.62$ $\left(1 \mathrm{H}, \mathrm{dd}, J=10.5,3.1 \mathrm{~Hz}, \mathrm{H}_{6 \mathrm{a}}\right), 4.55\left(1 \mathrm{H}, \mathrm{dd}, J=10.6,1.8 \mathrm{~Hz}, \mathrm{H}_{6 \mathrm{~b}}\right), 3.92-3.85\left(3 \mathrm{H}, \mathrm{m}, \mathrm{H}_{2}\right.$, $\left.\mathrm{H}_{3}, \mathrm{H}_{5}\right), 3.70\left(1 \mathrm{H}\right.$, app. t, $\left.J=9.7 \mathrm{~Hz}, \mathrm{H}_{4}\right) ;{ }^{13} \mathbf{C}$ NMR $\left(101 \mathrm{MHz} ; \mathrm{D}_{2} \mathrm{O}\right) \delta 95.0\left(\mathrm{~d}, J_{C, P}=5.6\right.$ $\left.\mathrm{Hz}, \mathrm{C}_{1}\right), 82.4\left(J_{C, F}=170.0 \mathrm{~Hz}, \mathrm{C}_{6}\right), 71.5\left(\mathrm{C}_{2}\right), 71.1\left(\mathrm{C}_{3}\right), 69.7\left(\mathrm{C}_{4}\right), 65.5\left(J_{C, F}=20.0 \mathrm{~Hz}, \mathrm{C}_{5}\right)$; ${ }^{31} \mathbf{P}\left\{{ }^{1} \mathbf{H}\right\}$ NMR $\left(162 \mathrm{MHz}, \mathrm{D}_{2} \mathrm{O}\right) \delta-1.81 ;{ }^{19} \mathbf{F}\left\{{ }^{1} \mathbf{H}\right\}$ NMR $\left(282 \mathrm{MHz}, \mathrm{D}_{2} \mathrm{O}\right) \delta-122.8$; HRMS $m / z$ (ESI-) Found: (M-H)- 261.0178, $\mathrm{C}_{6} \mathrm{H}_{10} \mathrm{FO}_{8} \mathrm{P}$ requires $(\mathrm{M}-\mathrm{H})^{-} 261.0181$.

\section{1,2,3,4-Tetra-O-acetyl-6-deoxy-6,6-difluoro- $\beta$-D-mannopyranose 16}

Dess-Martin periodinane $(749 \mathrm{mg}, 1.8 \mathrm{mmol}$ ) was added to a solution of 1,2,3,4-tetra- $O$ acetyl- $\beta$-D-mannose ${ }^{[30]}(513 \mathrm{mg}, 1.5 \mathrm{mmol})$ in dichloromethane $(5.0 \mathrm{~mL})$ and the reaction mixture was stirred at room temperature for $18 \mathrm{~h}$. Next, the reaction mixture was quenched with a saturated aqueous solution of $\mathrm{Na}_{2} \mathrm{~S}_{2} \mathrm{O}_{3}(5.0 \mathrm{~mL})$ and the aqueous layer was extracted with $\mathrm{CH}_{2} \mathrm{Cl}_{2}(3 \times 10 \mathrm{~mL})$. The combined organic phases were dried over $\mathrm{MgSO}_{4}$ and evaporated in vacuo. The crude aldehyde was then dissolved in dichloromethane $(10.0 \mathrm{~mL})$ and cooled to $0{ }^{\circ} \mathrm{C}$. DAST $(0.58 \mathrm{~mL}, 4.4 \mathrm{mmol})$ was added dropwise, after which the reaction mixture was allowed to warm to room temperature. After $18 \mathrm{~h}$, the reaction mixture was diluted with dichloromethane $(10 \mathrm{~mL})$ and quenched with a saturated aqueous solution of 
$\mathrm{NaHCO}_{3}(10.0 \mathrm{~mL})$. The aqueous layer was extracted with dichloromethane $(3 \times 10.0 \mathrm{~mL})$. The combined organic phases were dried over $\mathrm{MgSO}_{4}$ and evaporated in vacuo. The crude product was purified by flash column chromatography to yield 16 (45 mg, 8\%). $[\alpha]_{D}^{26}-19.5$ (c $\left.1.1, \mathrm{CHCl}_{3}\right) ;{ }^{1} \mathbf{H} \mathbf{N M R}\left(400 \mathrm{MHz}, \mathrm{CDCl}_{3}\right) \delta 5.92\left(1 \mathrm{H}, \mathrm{d}, J=1.2 \mathrm{~Hz}, \mathrm{H}_{1}\right), 5.89\left(1 \mathrm{H}, \mathrm{td}, J_{H, F}=\right.$ $\left.54.3 \mathrm{~Hz}, J_{H 6, H 5}=3.4 \mathrm{~Hz}, \mathrm{H}_{6}\right), 5.50-5.42(\mathrm{~m}, 2 \mathrm{H}), 5.18\left(\mathrm{dd}, J=9.6,3.3 \mathrm{~Hz}, 1 \mathrm{H}, \mathrm{H}_{3}\right), 3.90-3.81$ $(\mathrm{m}, 1 \mathrm{H}), 2.20\left(3 \mathrm{H}, \mathrm{s}, \mathrm{C}(\mathrm{O}) \mathrm{OCH}_{3}\right), 2.11\left(3 \mathrm{H}, \mathrm{s}, \mathrm{C}(\mathrm{O}) \mathrm{OCH}_{3}\right), 2.06\left(3 \mathrm{H}, \mathrm{s}, \mathrm{C}(\mathrm{O}) \mathrm{OCH}_{3}\right), 2.02$ $\left(3 \mathrm{H}, \mathrm{s}, \mathrm{C}(\mathrm{O}) \mathrm{OCH}_{3}\right) ;{ }^{13} \mathrm{C}$ NMR $\left(100 \mathrm{MHz}, \mathrm{CDCl}_{3}\right) \delta 170.2(\mathrm{C}=\mathrm{O}), 169.8(\mathrm{C}=\mathrm{O}), 169.5$ $(\mathrm{C}=\mathrm{O}), 168.4(\mathrm{C}=\mathrm{O}), 113.3\left(\mathrm{t}, J_{C, F}=246.0 \mathrm{~Hz}, \mathrm{C}_{6}\right), 90.1\left(\mathrm{C}_{1}\right), 73.2\left(\mathrm{t}, J_{C, F}=24.7 \mathrm{~Hz}, \mathrm{C}_{5}\right)$, $70.1\left(\mathrm{C}_{2}\right.$ or $\left.\mathrm{C}_{3}\right), 67.7\left(\mathrm{C}_{2}\right.$ or $\left.\mathrm{C}_{3}\right), 64.1\left(\mathrm{t}, J_{C, F}=1.2 \mathrm{~Hz}, \mathrm{C}_{4}\right), 20.84\left(3 \mathrm{H}, \mathrm{s}, \mathrm{C}(\mathrm{O}) \mathrm{OCH}_{3}\right), 20.79$ $\left(3 \mathrm{H}, \mathrm{s}, \mathrm{C}(\mathrm{O}) \mathrm{OCH}_{3}\right), 20.71\left(3 \mathrm{H}, \mathrm{s}, \mathrm{C}(\mathrm{O}) \mathrm{OCH}_{3}\right), 20.65\left(3 \mathrm{H}, \mathrm{s}, \mathrm{C}(\mathrm{O}) \mathrm{OCH}_{3}\right) ;{ }^{19} \mathbf{F}$ NMR $(376$ $\left.\mathrm{MHz}, \mathrm{CDCl}_{3}\right) \delta-126.6(\mathrm{ddd}, J=296.6,54.4,9.0 \mathrm{~Hz}, 1 \mathrm{~F}),-130.2(\mathrm{ddd}, J=296.8,54.6,10.6$ $\mathrm{Hz}, 1 \mathrm{~F}) ;{ }^{19} \mathbf{F}\left\{{ }^{1} \mathbf{H}\right\}$ NMR $\left(376 \mathrm{MHz}, \mathrm{CDCl}_{3}\right) \delta-126.6(\mathrm{~d}, J=296.4 \mathrm{~Hz}, 1 \mathrm{~F}),-130.2(\mathrm{~d}, J=$ $296.4 \mathrm{~Hz}, 1 \mathrm{~F}$ ); HRMS $\mathrm{m} / \mathrm{z}$ (ESI ${ }^{+}$) Found: $[\mathrm{M}+\mathrm{Na}]^{+} 391.0820, \mathrm{C}_{14} \mathrm{H}_{18} \mathrm{~F}_{2} \mathrm{NaO}_{9}$ requires $[\mathrm{M}+\mathrm{Na}]^{+} 391.0811$

\section{6-Deoxy-6,6-difluoro- $\alpha$-D-mannopyranosyl 1-phosphate bis ammonium salt 17}

16 (44 mg, $0.10 \mathrm{mmol}$ ) gave 17 (15 mg, $0.05 \mathrm{mmol}, 42 \%$ ).

${ }^{1} \mathbf{H}$ NMR $\left(600 \mathrm{MHz}, \mathrm{D}_{2} \mathrm{O}\right) \delta 6.07\left(1 \mathrm{H}, \mathrm{td}, J_{H, F}=55.3, J_{H, F}=54.9, J_{6,5}=5.5 \mathrm{~Hz}, \mathrm{H}_{6}\right), 5.28$ $\left(1 \mathrm{H}, \mathrm{dd}, J_{P, H}=8.5, J_{l, 2}=1.9 \mathrm{~Hz}, \mathrm{H}_{1}\right), 4.62-3.93\left(1 \mathrm{H}, \mathrm{m}, \mathrm{H}_{5}\right), 3.89-3.85\left(2 \mathrm{H}, \mathrm{m}, \mathrm{H}_{2}, \mathrm{H}_{3}\right), 3.76$ $\left(1 \mathrm{H}, \mathrm{t}, J=9.4 \mathrm{~Hz}, \mathrm{H}_{4}\right) ;{ }^{13} \mathbf{C} \mathbf{N M R}\left(101 \mathrm{MHz} ; \mathrm{D}_{2} \mathrm{O}\right) \delta 114.6\left(\mathrm{t}, J_{C, F}=182.0 \mathrm{~Hz}, \mathrm{C} 6\right), 95.0(\mathrm{~d}$, $\left.J_{C, P}=4.4 \mathrm{~Hz}, \mathrm{C}_{1}\right), 70.7\left(\mathrm{C}_{2}\right), 70.3\left(\mathrm{C}_{5}\right), 69.6\left(\mathrm{C}_{3}\right), 67.2\left(\mathrm{C}_{4}\right) ;{ }^{31} \mathbf{P}\left\{{ }^{1} \mathbf{H}\right\}$ NMR $\left(162 \mathrm{MHz}, \mathrm{D}_{2} \mathrm{O}\right)$ $\delta 1.75 ;{ }^{19} \mathbf{F}\left\{{ }^{1} \mathbf{H}\right\}$ NMR $\left(376 \mathrm{MHz}, \mathrm{D}_{2} \mathrm{O}\right) \delta-131.5(\mathrm{~d}, J=284.6 \mathrm{~Hz}, 1 \mathrm{~F}),-132.2(\mathrm{~d}, J=284.2$ $\mathrm{Hz}, 1 \mathrm{~F})$; HRMS $m / z$ (ESI-) Found: (M-H)- 279.0086, $\mathrm{C}_{6} \mathrm{H}_{9} \mathrm{~F}_{2} \mathrm{O}_{8} \mathrm{P}$ requires $(\mathrm{M}-\mathrm{H})^{-} 279.0087$.

\section{1,2,3,4-Tetra-O-acetyl-6-para-tolenesulfonyl- $\beta$-D-mannose}

To a stirred solution of 1,2,3,4-tetra-O-acetyl- $\beta$-D-mannose ${ }^{[30]}(250 \mathrm{mg}, 0.72 \mathrm{mmol}, 1.0$ equiv.) in anhydrous pyridine (4.8 mL) was added $p$ - TsCl (470 mg, $2.45 \mathrm{mmol}, 3.4$ equiv.). The reaction mixture was stirred for $7 \mathrm{~h}$ at room temperature, where TLC analysis (petroleum ether/EtOAc, 1/2) indicated complete conversion of starting material to a higher $\mathrm{R}_{\mathrm{f}}$ value spot. Distilled water was added to the reaction mixture $(10 \mathrm{~mL})$ and stirred for $10 \mathrm{~min}$ before extraction with chloroform $(30 \mathrm{~mL})$. The organic layer was washed with saturated aqueous $\mathrm{NaHCO}_{3}$ solution $(2 \times 20 \mathrm{~mL})$, distilled water $(2 \times 20 \mathrm{~mL})$ and brine $(20 \mathrm{~mL})$ then the combined aqueous layers were re-extracted with chloroform $(25 \mathrm{~mL})$. The combined organic layers were dried $\left(\mathrm{MgSO}_{4}\right)$, filtered and concentrated under reduced pressure. Drying under high vacuum afforded the title compound as a colourless syrup (340 $\mathrm{mg}, 0.67 \mathrm{mmol}, 93 \%$ ). $\mathrm{R}_{\mathrm{f}} 0.82$ (petroleum ether/EtOAc, 1/2); $[\alpha]_{D}^{26}-6.70$ (c 1.65, $\left.\mathrm{CHCl}_{3}\right) ;{ }^{1} \mathbf{H}$ NMR $(400 \mathrm{MHz}$, $\left.\mathrm{CDCl}_{3}\right) \delta_{\mathrm{H}}$ 7.78-7.74 (2H, m, Ar- $\left.H\right), 7.33(2 \mathrm{H}, \mathrm{d}, J=8.0 \mathrm{~Hz}, 2 \times \operatorname{Ar}-H), 7.30-7.27(1 \mathrm{H}, \mathrm{m}$, $\operatorname{Ar}-H), 5.79\left(1 \mathrm{H}, \mathrm{d}, J=1.2 \mathrm{~Hz}, \mathrm{H}_{1}\right), 5.42\left(1 \mathrm{H}, \mathrm{dd}, J=3.2,1.2 \mathrm{~Hz}, \mathrm{H}_{2}\right), 5.20(1 \mathrm{H}$, appt, $J=9.8$ $\left.\mathrm{Hz}, \mathrm{H}_{4}\right), 5.08\left(1 \mathrm{H}, J=9.9,3.3 \mathrm{~Hz}, \mathrm{H}_{3}\right), 4.13\left(2 \mathrm{H}\right.$, app. d, $\left.J=4.3 \mathrm{~Hz}, \mathrm{H}_{6 \mathrm{a}+\mathrm{b}}\right), 3.79(1 \mathrm{H}, \mathrm{dt}, J=$ 9.6, $\left.4.3 \mathrm{~Hz}, \mathrm{H}_{5}\right), 2.43\left(3 \mathrm{H}, \mathrm{s}, \mathrm{CH}_{3}\right), 2.16\left(3 \mathrm{H}, \mathrm{s}, \mathrm{C}(\mathrm{O}) \mathrm{OCH}_{3}\right), 2.06\left(3 \mathrm{H}, \mathrm{s}, \mathrm{C}(\mathrm{O}) \mathrm{OCH}_{3}\right), 2.00$ $\left(3 \mathrm{H}, \mathrm{s}, \mathrm{C}(\mathrm{O}) \mathrm{OCH}_{3}\right), 1.98\left(3 \mathrm{H}, \mathrm{s}, \mathrm{C}(\mathrm{O}) \mathrm{OCH}_{3}\right) ;{ }^{13} \mathbf{C} \mathbf{N M R}\left(100 \mathrm{MHz}, \mathrm{CDCl}_{3}\right) \delta 170.3(\mathrm{C}=\mathrm{O})$, $169.9(\mathrm{C}=\mathrm{O}), 169.7(\mathrm{C}=\mathrm{O}), 168.3(\mathrm{C}=\mathrm{O}), 149.9(\mathrm{Ar}-\mathrm{C}), 145.2(\mathrm{Ar}-\mathrm{C}), 136.1(\mathrm{Ar}-\mathrm{C}), 132.6$ (Ar-C), $130.0(\mathrm{Ar}-C), 128.2(\mathrm{Ar}-C), 90.3\left(\mathrm{C}_{1}\right), 70.9\left(\mathrm{C}_{5}\right), 70.5\left(\mathrm{C}_{4}\right), 68.1\left(\mathrm{C}_{3}\right), 67.6\left(\mathrm{C}_{2}\right), 65.8$ $\left(\mathrm{C}_{6}\right), 21.8\left(\mathrm{CH}_{3}\right), 20.8\left(\mathrm{C}(\mathrm{O}) \mathrm{CH}_{3}\right), 20.7\left(2 \times \mathrm{C}(\mathrm{O}) \mathrm{CH}_{3}\right), 20.6\left(\mathrm{C}(\mathrm{O}) \mathrm{CH}_{3}\right) ; \mathbf{H R M S ~ m} / z\left(\mathrm{ESI}^{+}\right)$ Found: $(\mathrm{M}+\mathrm{Na})^{+} 525.1073, \mathrm{C}_{21} \mathrm{H}_{26} \mathrm{O}_{12} \mathrm{SNa}$ requires $[\mathrm{M}+\mathrm{Na}]^{+}$, 525.1043. Previously reported data were for the $\alpha$-anomer. ${ }^{[36]}$

\section{1,2,3,4-Tetra-O-acetyl-6-azido-6-deoxy- $\beta$-D-mannose 18}

To a stirred solution of 1,2,3,4-tetra-O-acetyl-6-para-tolenesulfonyl- $\beta$-D-mannose (200 mg, $0.39 \mathrm{mmol}, 1.0$ equiv. $)$ in DMF (3.9 mL) was added successively 15-crown-5 ether $(0.23 \mathrm{~mL}$, 
1.20 mmol, 3.0 equiv.) and $\mathrm{NaN}_{3}(78 \mathrm{mg}, 1.20 \mathrm{mmol}, 3.0$ equiv.). The reaction mixture was heated to $60{ }^{\circ} \mathrm{C}$ and stirred for $24 \mathrm{~h}$, whereby TLC analysis (toluene/EtOAc, 4/1) indicated conversion of starting material to a higher $\mathrm{R}_{\mathrm{f}}$ value spot. The reaction mixture was cooled to room temperature, poured onto distilled water $(20 \mathrm{~mL})$ and extracted with EtOAc $(25 \mathrm{~mL})$. The organic layer was washed successively with $10 \%$ aqueous $\mathrm{Na}_{2} \mathrm{~S}_{2} \mathrm{O}_{3}$ solution, distilled water and brine $(20 \mathrm{~mL}$ each) then the aqueous layers were re-extracted with EtOAc $(25 \mathrm{~mL})$. The combined organic layers were dried $\left(\mathrm{MgSO}_{4}\right)$, filtered and concentrated under reduced pressure. Purification by silica gel column chromatography, eluting with toluene/EtOAc (5/1, 4/1) afforded 18 as a clear colourless oil $(31 \mathrm{mg}, 83 \mu \mathrm{mol}, 32 \%) . \mathrm{R}_{\mathrm{f}} 0.33$ (toluene/acetone, 4/1); $[\alpha]_{D}^{26}-13.5\left(c\right.$ 1.55, $\left.\mathrm{CHCl}_{3}\right){ }^{1} \mathbf{H}$ NMR $\left(400 \mathrm{MHz}, \mathrm{CDCl}_{3}\right) \delta 5.86\left(1 \mathrm{H}, \mathrm{d}, J=1.2 \mathrm{~Hz}, \mathrm{H}_{1}\right)$, $5.47\left(1 \mathrm{H}, \mathrm{dd}, J=3.2,1.1 \mathrm{~Hz}, \mathrm{H}_{2}\right), 5.25\left(1 \mathrm{H}\right.$, app. t, $\left.J=9.8 \mathrm{~Hz}, \mathrm{H}_{4}\right), 5.11(1 \mathrm{H}, \mathrm{dd}, J=10.0$, $\left.3.2 \mathrm{~Hz}, \mathrm{H}_{3}\right), 3.78-.3 .72\left(1 \mathrm{H}, \mathrm{m}, \mathrm{H}_{5}\right), 3.38\left(2 \mathrm{H}\right.$, app. qd, $\left.J=13.4,4.5 \mathrm{~Hz}, \mathrm{H}_{6 \mathrm{a}}, \mathrm{H}_{6 \mathrm{~b}}\right), 2.21(3 \mathrm{H}$, s, $\left.\mathrm{C}(\mathrm{O}) \mathrm{CH}_{3}\right), 2.09\left(3 \mathrm{H}, \mathrm{s}, \mathrm{C}(\mathrm{O}) \mathrm{CH}_{3}\right), 2.05\left(3 \mathrm{H}, \mathrm{s}, \mathrm{C}(\mathrm{O}) \mathrm{CH}_{3}\right), 1.99\left(3 \mathrm{H}, \mathrm{s}, \mathrm{C}(\mathrm{O}) \mathrm{CH}_{3}\right) ;{ }^{13} \mathrm{C}$ NMR $\left(100 \mathrm{MHz}, \mathrm{CDCl}_{3}\right) \delta 170.3(\mathrm{C}=\mathrm{O}), 169.9(\mathrm{C}=\mathrm{O}), 169.8(\mathrm{C}=\mathrm{O}), 168.5(\mathrm{C}=\mathrm{O}), 90.3$ $\left(\mathrm{C}_{1}\right), 74.5\left(\mathrm{C}_{5}\right), 70.7\left(\mathrm{C}_{3}\right), 68.2\left(\mathrm{C}_{2}\right), 66.5\left(\mathrm{C}_{4}\right), 50.8\left(\mathrm{C}_{6}\right), 20.9\left(\mathrm{C}(\mathrm{O}) \mathrm{CH}_{3}\right), 20.8(2 \times$ $\left(\mathrm{C}(\mathrm{O}) \mathrm{CH}_{3}\right), 20.6\left(\mathrm{C}(\mathrm{O}) \mathrm{CH}_{3}\right)$; HRMS $m / z\left(\mathrm{ESI}^{+}\right)$Found: $(\mathrm{M}+\mathrm{Na})^{+} 396.1039, \mathrm{C}_{14} \mathrm{H}_{19} \mathrm{~N}_{3} \mathrm{O}_{9} \mathrm{Na}$ requires $[\mathrm{M}+\mathrm{Na}]^{+}, 396.1019$. Previously reported data were for the $\alpha$-anomer. ${ }^{[36]}$

\section{6-Azido-6-deoxy- $\alpha$-D-mannose 1-phosphate bis ammonium salt 19}

18 (100 mg, $0.27 \mathrm{mmol}$ ) gave 19 (25 mg, $0.14 \mathrm{mmol}, 65 \%)$.

${ }^{1} \mathbf{H}$ NMR $\left(400 \mathrm{MHz}, \mathrm{D}_{2} \mathrm{O}\right) \delta 5.07\left(1 \mathrm{H}, \mathrm{dd}, J_{P, H}=8.4, J_{1,2}=1.9 \mathrm{~Hz}, \mathrm{H}_{1}\right), 3.72-3.67(3 \mathrm{H}, \mathrm{m}$, $\left.\mathrm{H}_{5}, \mathrm{H}_{2}, \mathrm{H}_{4}\right), 3.50-3.45\left(1 \mathrm{H}, \mathrm{m}, \mathrm{H}_{6 \mathrm{a}}\right), 3.42-3.37\left(2 \mathrm{H}, \mathrm{m}, \mathrm{H}_{3} \mathrm{H}_{6 \mathrm{~b}}\right) ;{ }^{13} \mathbf{C}$ NMR $\left(101 \mathrm{MHz}, \mathrm{D}_{2} \mathrm{O}\right) \delta$ $94.9\left(\mathrm{C}_{1}\right), 72.4,71.1,69.7\left(\mathrm{C}_{2}, \mathrm{C}_{4}\right.$ or $\left.\mathrm{C}_{5}\right), 67.2\left(\mathrm{C}_{3}\right), 51.0\left(\mathrm{C}_{6}\right) ;{ }^{31} \mathbf{P}\left\{{ }^{1} \mathbf{H}\right\}$ NMR $(162 \mathrm{MHz}$, $\left.\mathrm{D}_{2} \mathrm{O}\right) \delta$ 1.36; HRMS $m / z$ (ESI-) Found: $(\mathrm{M}-\mathrm{H})^{-} 284.0288, \mathrm{C}_{6} \mathrm{H}_{11} \mathrm{~N}_{3} \mathrm{O}_{8} \mathrm{P}$ requires $[\mathrm{M}-\mathrm{H}]^{-}$ 284.0284. Data previously reported for the bis-triethylammonium salt. ${ }^{[37]}$

\section{1,2,3,4-Tetra-O-acetyl-6-S-acetyl-6-deoxy- $\beta$-D-mannose 20}

To a stirred solution of 1,2,3,4-Tetra-O-acetyl-6-chloro-6-deoxy- $\beta$-D-mannose (58 mg, 0.16 mmol, 1.0 equiv.) in anhydrous DMF $(1.6 \mathrm{ml})$ was added potassium thioacetate $(54 \mathrm{mg}, 0.48$ mmol, 3.0 equiv.). The reaction mixture was heated to $75{ }^{\circ} \mathrm{C}$ for 24 hours, where TLC analysis (petroleum ether/EtOAc, 2/1) indicated formation of a similar $\mathrm{R}_{\mathrm{f}}$ value product to the starting material). The reaction mixture was cooled to room temperature then poured onto water $(10 \mathrm{~mL})$ and extracted with EtOAc $(20 \mathrm{~mL})$. The organic layer was washed successively with saturated aqueous $\mathrm{NaHCO}_{3}$ solution, water and brine $(15 \mathrm{~mL}$ each) then the aqueous layers were re-extracted with EtOAc $(20 \mathrm{~mL})$. The combined organic layers were dried $\left(\mathrm{MgSO}_{4}\right)$, filtered and concentrated under reduced pressure. Purification by silica gel column chromatography, eluting with petroleum ether/EtOAc (2/1) afforded $\mathbf{2 0}$ as a clear orange oil (55 mg, $0.14 \mathrm{mmol}, 88 \%$ ). $\mathrm{R}_{\mathrm{f}} 0.38$ (petroleum ether/EtOAc, 2/1); $[\alpha]_{D}^{26}-7.27$ (c $\left.1.45, \mathrm{CHCl}_{3}\right) ;{ }^{1} \mathbf{H}$ NMR $\left(400 \mathrm{MHz}, \mathrm{CDCl}_{3}\right) \delta 5.80\left(1 \mathrm{H}, \mathrm{s}, \mathrm{H}_{1}\right), 5.45-5.43\left(1 \mathrm{H}, \mathrm{m}, \mathrm{H}_{2}\right), 5.18$ $\left(1 \mathrm{H}\right.$, app. t $\left.J=9.8, \mathrm{H}_{4}\right), 5.07\left(1 \mathrm{H}, \mathrm{dd}, J=9.9,3.2 \mathrm{~Hz}, \mathrm{H}_{3}\right), 3.68(1 \mathrm{H}$, ddd, $J=9.8,7.5,2.7$ $\left.\left.\mathrm{Hz}, \mathrm{H}_{5}\right), 3.28,1 \mathrm{H}, \mathrm{dd}, J=14.3,2.5 \mathrm{~Hz}, \mathrm{H}_{6 \mathrm{a}}\right), 3.03\left(1 \mathrm{H}, \mathrm{dd}, J=14.3,7.4 \mathrm{~Hz}, \mathrm{H}_{6 \mathrm{~b}}\right), 2.32(3 \mathrm{H}, \mathrm{s}$, $\left.\mathrm{SCOCH}_{3}\right), 2.19\left(3 \mathrm{H}, \mathrm{s}, \mathrm{COCH}_{3}\right), 2.10\left(3 \mathrm{H}, \mathrm{s}, \mathrm{COCH}_{3}\right), 2.08\left(3 \mathrm{H}, \mathrm{s}, \mathrm{COCH}_{3}\right), 1.98(3 \mathrm{H}, \mathrm{s}$, $\left.\mathrm{COCH}_{3}\right) ;{ }^{13} \mathrm{C}$ NMR $\left(100 \mathrm{MHz}, \mathrm{CDCl}_{3}\right) \delta 194.9(\mathrm{C}=\mathrm{O}), 170.3(\mathrm{C}=\mathrm{O}), 170.1(\mathrm{C}=\mathrm{O}), 169.9$ $(\mathrm{C}=\mathrm{O}), 168.4(\mathrm{C}=\mathrm{O}), 90.5\left(\mathrm{C}_{1}\right), 74.7\left(\mathrm{C}_{5}\right), 70.7\left(\mathrm{C}_{3}\right), 68.4\left(\mathrm{C}_{2}\right), 67.8\left(\mathrm{C}_{4}\right), 30.5\left(\mathrm{SCOCH}_{3}\right)$, $30.2\left(\mathrm{C}_{6}\right), 20.9\left(2 \mathrm{C}, 2 \times \mathrm{COCH}_{3}\right), 20.8\left(\mathrm{COCH}_{3}\right), 20.6\left(\mathrm{COCH}_{3}\right)$; HRMS $m / z\left(\mathrm{ESI}^{+}\right)$Found: $(\mathrm{M}+\mathrm{Na})^{+} 424.0832 \mathrm{C}_{16} \mathrm{H}_{22} \mathrm{O}_{10} \mathrm{SNa}$, requires $[\mathrm{M}+\mathrm{Na}]^{+} 424.0832$.

6-Deoxy-6-thio- $\alpha$-D-mannose 1-phosphate bis ammonium salt 21

20 (100 mg, $0.25 \mathrm{mmol}$ ) gave 21 (30 mg, $0.10 \mathrm{mmol}, 43 \%$ ). 
${ }^{1} \mathbf{H}$ NMR $\left(400 \mathrm{MHz}, \mathrm{D}_{2} \mathrm{O}\right) \delta 5.34\left(\mathrm{~d}, J_{P, H}=8.1 \mathrm{~Hz}, \mathrm{H}_{1}\right.$ (1-phosphate disulfide), $5.16\left(\mathrm{~d}, J_{1,2}=\right.$ $1.3 \mathrm{~Hz}$ (1-phosphate-hemiacetal mixed disulfide), ratio $=2.5 / 1 ;{ }^{31} \mathbf{P}\left\{{ }^{1} \mathbf{H}\right\} \mathbf{N M R}(162 \mathrm{MHz}$, $\left.\mathrm{D}_{2} \mathrm{O}\right) \delta 0.38$ (1P, s, 1-phosphate disulfide); HRMS $m / z$ (ESI $\left.{ }^{-}\right)$of the disulfide: Found: (M-H) 548.9910, $\mathrm{C}_{12} \mathrm{H}_{23} \mathrm{O}_{16} \mathrm{P}_{2} \mathrm{~S}_{2}$ requires [M-H] ${ }^{-}$548.9908; of the mixed disulfide: Found: $(\mathrm{M}-\mathrm{H})^{-}$ $469.0239, \mathrm{C}_{12} \mathrm{H}_{22} \mathrm{O}_{13} \mathrm{PS}_{2}$ requires [M-H] $]^{-} 469.0240$.

\section{DFT calculations}

The geometry optimisations were performed with Gaussian $16^{[38]}$ software using restricted density functional theory. The B3LYP ${ }^{[39]}$ functional hybrid method was employed and the $6-311+\mathrm{G}(2 \mathrm{df}, \mathrm{p})^{[40,41]}$ with diffused basis set was used for the geometry optimisation and frequency analysis in vacuum. The normal modes revealed no imaginary frequencies indicating that they represent minima on the potential energy surface.

\section{References:}

[1] Pale, P. Whitesides, G. M. J. Org. Chem. 1991, 56, 4547-4549.

[2] Fujita, S.; Oka, N.; Matsumura, F.; Wada, T. J. Org. Chem. 2011, 76, 2648-2659.

[3] Shashkov, A.S.; Streshinskaya, G.M.; Tulskaya, E.M.; Senchenkova, S.N.; Baryshnikova, L.M.; Dmitrenok, A.S.; Ostash, B.E.; Fedorenko, V.A. Int. J. Gen. Mol. Microbiol. 2016, 109, 923-936.

[4] Qi, X.; Ma, M.; Wang, L.; Zhang, Y.; Jiang, R.; Rong, B.; Li, Y. Biochem. Biophys. Res. Commun. 2015, 465, 113-118.

[5] a) Ahmadipour, S.; Miller, G. J. Carbohydr. Res. 2017, 451, 95-109 b) Ahmadipour, S.; Beswick, L.; Miller, G. J. Carbohydr. Res. 2018, 469, 38-47.

[6] Wagner, G. K.; Pesnot, T.; Field, R. A. Nat. Prod. Rep. 2009, 26, 1172-1194.

[7] Gantt, R. W.; Peltier-Pain, P.; Singh, S.; Zhou, M.; Thorson, J. S. Proc. Natl. Acad. Sci. U.S.A. 2013, 110, 7648-7653.

[8] a) Pergolizzi, G.; Kuhaudomlarp, S.; Kalita, E.; Field, R. A. Protein Pept. Lett. 2017, 24, 696-709 b) Nakai, H.; Kitaoka, M.; Svensson, B.; Ohtsubo, K. Current Opinion in Chemical Biology 2013, 17, 301-309 and refs. Therein c) E. C. O'Neill and R. A. Field, Carbohydr. Res., 2015, 403, 23-37.

[9] Sernee, M. F.; Ralton, J. E.; Nero, T. L.; Sobala, L. F.; Kloehn, J.; Vieira-Lara, M. A.; Cobbold, S. A.; Stanton, L.; Pires, D. E. V.; Hanssen, E.; Males, A.; Ward, T.; Bastidas, L. M.; van der Peet, P. L.; Parker, M. W.; Ascher, D. B.; Williams, S. J.; Davies, G. J.; McConville, M. J. Cell Host and Microbe 2019, 26, 385-399.e9.

[10] De Groeve, M.R.; Depreitere, V.; Desmet, T.; Soetaert, W. Biotechnol. Lett. 2009, 31, $1873-1877$.

[11] Wen, L.; Huang, K.; Wei, M.; Meisner, J.; Liu, Y.; Garner, K.; Zang, L.; Wang, X.; Li, X.; Fang, J.; Zhang, H.; Wang, P. G. Angew. Chem. Int. Ed. Engl. 2015, 54, 12654-12658.

[12] Yu, H.; Chen, X. Org. Biomol. Chem. 2016, 14, 2809-2818.

[13] Wildberger, P.; Pfeiffer, M.; Brecker, L.; Nidetzky, B. Angew. Chem. Int. Ed. 2015, 54, $15867-15871$.

[14] Huang, K.; Parmeggiani, F.; Pallister, E.; Huang, C.-J.; Liu, F.-F.; Li, Q.; Birmingham, W. R.; Both, P.; Thomas, B.; Liu, L.; Voglmeir, J.; Flitsch, S. L. ChemBioChem 2018, 19, 388-394.

[15] Keenan, T.; Mills, R.; Pocock, E.; Budhadev, D.; Parmeggiani, F.; Flitsch, S.; Fascione, M. Carbohydr. Res. 2019, 472, 132-137.

[16] Mizanur, R. M.; Pohl, N. L. B. Org. Biomol. Chem. 2009, 7, 2135-2139.

[17] Li, T.; Tikad, A.; Pan, W.; Vincent, S. P. Org. Lett. 2014, 16, 5628-5631.

[18] a) Errey, J.C.; Mann, M.C.; Fairhurst, S.A.; Hill, L.; McNeil, M.R.; Naismith, J.H.; Percy, J.M.; Whitfield, C.; Field, R.A. Org. Biomol. Chem., 2009, 7, 1009-16 b) Errey, J.C.; 
Mukhopadhyay, B.; Kartha, K.P.R.; Field, R.A. Chem. Commun., 2004, 2706-7 c) N'Go, I.; Golten, S.; Ardá, A.; Cañada, J.; Jiménez-Barbero, J.; Linclau, B.; Vincent, S.P. Chem. Eur. J., 2014, 20, 106-12.

[19] Ahmadipour, S.; Pergolizzi, G.; Rejzek, M.; Field, R. A.; Miller, G. J. Org. Lett. 2019, $21,4415-4419$.

[20] Dimitriou, E.; Miller, G. J. Org. Biomol. Chem. 2019, 17, 9321-35.

[21] Beswick, L.; Ahmadipour, S.; Dolan, J.P.; Rejzek, M.; Field, R.A.; Miller, G.J. Carbohydr. Res., 2019, 485, 107819.

[22] Schultz, V. L.; Zhang, X.; Linkens, K.; Rimel, J.; Green, D. E.; DeAngelis, P. L.; Linhardt, R. J. J. Org. Chem. 2017, 82, 2243-2248.

[23] Masuko, S.; Bera, S.; Green, D. E.; Weïwer, M.; Liu, J.; DeAngelis, P. L.; Linhardt, R. J. J. Org. Chem. 2012, 77, 1449-1456.

[24] Ko, H.; Das, A.; Carter, R. L.; Fricks, I. P.; Zhou, Y.; Ivanov, A. A.; Melman, A.; Joshi, B. V.; Kovác, P.; Hajduch, J.; Kirk, K. L.; Harden, T. K.; Jacobson, K. A. Bioorg. Med. Chem. 2009, 17, 5298-5311.

[25] Weïwer, M.; Sherwood, T.; Green, D. E.; Chen, M.; DeAngelis, P. L.; Liu, J.; Linhardt, R. J. J. Org. Chem. 2008, 73, 7631-7637.

[26] Watt, G. M.; Flitsch, S. L.; Fey, S.; Elling, L.; Kragl, U. Tetrahedron: Asymmetry 2000, $11,621-628$.

[27] Campbell, R. E.; Tanner, M. E. J. Org. Chem. 1999, 64, 9487-9492.

[28] MacDonald, D. L. J. Org. Chem. 1962, 27, 1107-1109.

[29] Doores, K.J.; Fulton, Z.; Hong, V.; Patel, M.K.; Scanlan, C.N.; Wormald, M.R.; Finn, M.G.; Burton, D.R.; Wilson, I.A.; Davis, B.G. Proc. Natl. Acad. Sci., 2010, 107, 17107-12.

[30] Beswick, L.; Miller, G. Molbank 2017, 2017, M947.

[31] Szabó, P.; Szabó, L. J. Chem. Soc., 1961, 448-57.

[32] Khan, S.H.; Jain, R.K.; Abbas, S.A.; Matta, K.L. Carbohydr. Res., 1989, 198, 259-73.

[33] Zhang, L.; Zhang, W.; Liu, J.; Hu, J. J. Org. Chem. 2009, 74, 2850-53.

[34] Beale, T.M.; Moon, P.J.; Taylor, M.S. Org. Lett., 2014, 16, 3604-7.

[35] Pallanca, J.E.; Turner, N.J. J. Chem. Soc., Perkin Trans. 1, 1993, 45, 3017-22.

[36] Kong, D.; Itzstein, von, M. Carbohydr. Res. 1997, 305, 323-329.

[37] Marchesan, S.; Macmillan, D. Chem. Commun. 2008, 4321-4323.

[38] M. J. Frisch, G. W. Trucks, H. B. Schlegel, G. E. Scuseria, M. A. Robb, J. R. Cheeseman, G. Scalmani, V. Barone, G. A. Petersson, H. Nakatsuji, X. Li, M. Caricato, A. V. Marenich, J. Bloino, B. G. Janesko, R. Gomperts, B. Mennucci, H. P. Hratchian, J. V. Ortiz, A. F. Izmaylov, J. L. Sonnenberg, D. Williams-Young, F. Ding, F. Lipparini, F. Egidi, J. Goings, B. Peng, A. Petrone, T. Henderson, D. Ranasinghe, V. G. Zakrzewski, J. Gao, N. Rega, G. Zheng, W. Liang, M. Hada, M. Ehara, K. Toyota, R. Fukuda, J. Hasegawa, M. Ishida, T. Nakajima, Y. Honda, O. Kitao, H. Nakai, T. Vreven, K. Throssell, J. A. Montgomery, Jr., J. E. Peralta, F. Ogliaro, M. J. Bearpark, J. J. Heyd, E. N. Brothers, K. N. Kudin, V. N. Staroverov, T. A. Keith, R. Kobayashi, J. Normand, K. Raghavachari, A. P. Rendell, J. C. Burant, S. S. Iyengar, J. Tomasi, M. Cossi, J. M. Millam, M. Klene, C. Adamo, R. Cammi, J. W. Ochterski, R. L. Martin, K. Morokuma, O. Farkas, J. B. Foresman, and D. J. Fox. Gaussian 16, Revision C.01. Gaussian, Inc.: Wallingford CT, 2019.

[39] a) Becke, A.D. J.Chem.Phys., 1993, 98, 5648-5652 b) Becke, A.D. Phys.Rev., A, 1988, 38, 3098.

[40] Frisch, M.J.; Pople, J.A.; Binkley, J.S. J. Chem. Phys., 1984, 80, 3265-3269.

[41] Hariharan, P.C.; Pople, J.A. Theoret. Chim. Acta, 1973, 28, 213-222. 